\title{
XXIV.
}

Aus der chirurgisehen Universitäts-Poliklinik (vormals

Prof. Friedrich) zu Leipzig.

\section{Experimentelle Beiträge zur Kenntnis des Transsudations- vorganges am Bauchfell, sowie zur Kenntnis einiger klinisch wichtigen Eigenschaften des Glycerinum officinale.}

\author{
Von \\ Dr. med. Gustav Schrader, \\ Assistenzarzt.
}

Seit der 1863 erschienenen Arbeit von Recklinghausens "Zur Fettresorption" ${ }^{1)}$ sind auf dem Wege experimenteller Forschung über die Funktionen und Eigenschaften des Peritoneums viele wertvolle Ergebnisse gewonnen worden, deren Kenntnis und Verwertung für eine begründete therapeutische Nutzanwendung bei Erkrankungen des Bauchfelles, namentlich bei endzïndlich peritonitischen Affektionen, von grober Tragweite geworden sind. Das Verdienst, nach dieser Richtung hin fördernd und anregend gewirkt zu haben, gebührt vor allem Wegner ${ }^{2}$ ), dessen inhaltsreiche Arbeit aus dem Jahre 1876 als Basis für die meisten späteren Untersuchungen anzusehen ist.

Wegner hat an der Hand des Tierexperimentes die „plyysiologisehen Einrichtungen in der Bauchböhle" dargetan, hat zuerst auf die Bedeutung der großen Flächenausdehnung, auf die enorme Resorptions- und Transsudationsfähigkeit, sowie auf die sogenannte "Plastizität" des Peritoneums mit Nachdruck hingewiesen und aus diesen Eigenschaften schon wichtige therapeutische Schlubfolgerungen für die Peritonitis abgeleitet.

Die nach ihm entstandenen Arbeiten sind nicht immer so universell gehalten; sie greifen meist nur eine Eigensehaft des Peritoneums heraus, um sie des Näheren zu studieren. So hat be-

1) v. Recklinghausen, Zur Fettresorption. Virchows Archiv. 24. Bd.

2) Wegnor, Chirurgische Bemerkungen über die Peritonealhöhle mit besonderer Berücksichtigung der Ovariotomie. Archiv f. klin. Chirurgie. 20. Bd.

Deutsehe Zeitschrift f. Chirurgie. LXX. Bd. 
sonders der Resorptionsvorgang allgemeines Interesse erweckt, und durch zahlreiche Arbeiten ist unsere Kenntnis über das Zustandekommen, die Gesetze und die wirksamen Kräfte, über Wesen und Bedeutung, über Intensität und Schnelligkeit der peritonealen Resorption bei intakter sowie bei gesehädigter Serosa bereichert und vertieft worden.

Die Ergebnisse der hier in Betracht kommenden experimentellen Studien lassen sich, soweit sie für pathologis che Verhältnisse des Bauchfells bedeutsam erscheinen, kurz folgendermaßen zusammenfassen: Der großen Ausdehnung wegen ist die Resorptionskraft des Peritoneums eine so gewaltige, daß in einer Stunde das Versuchstier 3,3-8 Proz. seines Körpergewichtes an Flüssigkeit aufzusaugen vermag (Wegner); sie ist an Intensität der subkutanen Resorption weit überlegen und rangiert in bezug auf Schnelligkeit der Wirkung unmittelbar hinter der direkten Injektion ins Blut (Wegner, Klapp 1)). $\mathrm{Ob}$ durch eine gesteigerte Peristaltik eine Resorptionsbeschleunigung zuwege kommt, scheint noch streitig; so sebr sie auch a priori wahrscheinlich ist. Während Schnitzler und Ewald ${ }^{2}$ ) eine Beschleunigung in ihren Versuchen nicht erzielten, mußten neuerdings Clairmont und $\mathrm{Haberer}{ }^{3}$ ) diese Frage in bejahendem Sinne beantworten. Die Herabsetzung der Peristaltik, sowie die Einwirkung der Kälte sind imstande, die Resorption einzuschränken. O. Cohnheim ${ }^{4}$ ), welcher bei $K \ddot{u} h n$ arbeitete, bezeichnet die Resorptionskraft des Bauchfells "wenn es in krankhaften Zuständen dazu gezwungen wird", als eine beschränkte. Bei bakterieller Peritonitis ist die Resorption nach Schnitzler und Ewald eine verlangsamte, nach Clairmont und Haberer anfangs eine beschleunigte und erst später eine verzögerte; ferner ist sie verlangsamt bei intraabdominaler Einspritzung von Bakterientoxinen- und Proteinen (Schnitzler und Ewald) und nach Schädigung der Serosa mittelst chemischer Agentien (Hamburger ${ }^{5}$ ).

Die praktisch gleichwichtige Frage nach den Wegen, auf denen

1) Klapp, Über Bauchfellresorption. Mitteilungen aus den Grenzgebieten der Medizin und Chirurgie. 10. Bd. 1902.

2) Schnitzler und Ewald, Zur Kenntnis der peritonealen Resorption. Deutsche Zeitschr. f. Chirurgie 41. Bd. 1895.

3) Clairmont und Haberer, Über das Verhalten des gesunden und veränderten tierischen Peritoneums. Wiener klin. Wochenschr. Nr. 45. 1902.

4) 0 . Cohaheim, Über die Resorption im Dünndarm und in der Bauchhöhle. Zeitschr. f. Biologie. 37. Bd. 1898.

5) Hamburger, Über die Regelung der osmotischen Spannkraft von Flüssigkeiten in Bauch- und Pericardialhöhle. Archiv f. Physiologie. Jahrg. 1895. 
sich diese Resorption vollzieht, ist namentlich durch Heiden hain ${ }^{1}$, Orlow ${ }^{2}$, Hamburger, Notkin und Becks), Dubar und Rem ${ }^{4}$ ), Starling und Tubby ${ }^{5}$ ) dahin entschieden, daß die Funktion der Aufsaugung aus der Bauchhöhle nicht, wie man früher annahm, den Lymphbahnen, speziell dem Centrum tendineum des Zwerchfells, sondern im wesentlichen den Blutkapillaren an allen Stellen des Peritoneums zufällt.

Auf diesen Ergebnissen fußend hat man die Resorptionskraft des Bauchfells auch als wirksame Schutzeinrichtung des Organismus gegen die peritonitische Infektion gedeutet und gewürdigt. Durch viele experimentelle Untersuchungen ist es jetzt als erwiesen zu betrachten, daß das Peritoneum dank dieser Fähigkeit eine intraabdominale Injektion von beträchtlichen, in indifferenter Flüssigkeit suspendierten Bakterienmengen verträgt, ohne mit einer Entzïndung zu antworten. Tavel und $\mathrm{Lanz}^{6}$ ) gehen auf Grund ihrer eingehenden Untersuchungen sogar soweit, den Satz aufzustellen: "Eine bakterielle primäre Peritonitis gibt es nicht, weil die normale Serosa durch ihre Resorptionskraft die geimpften Bakterien leicht resorbiert oder überhaupt sich von ihnen nicht angreifen läßt" (S. 143). Wenn nun auch diese Worte nicht eine bedingungslose Zustimmung verdienen, da auch bei Nichtiiberschreitung der Resorptionsfähigkeit eines intakten normalen Peritoneums tatsächlich eine primäre Peritonitis durch Injektion von vollvirulentem Infektionsmaterial zu erzielen ist (Pawlowsky ), Burginsky ${ }^{8}$ ), Wallgreen ${ }^{9}$ ), so verdient doch die grobe $\mathrm{Be}$ deutung der peritonealen Resorption, die eben in einer schnellen Fortschaffung der Bakterien ihren Ausdruck findet, eine volle Würdigung. indem tatsächlich das Zustandekommen einer allgemeinen Peritonitis

1) Heidenhein, Bemerkungen und Versuche betreffs der Resorption in der Banchhöhle. Arehiv f. d. gesamte Physiologie. 62. Bd. 1895.

2) Orlow, Einige Versuche über die Resorption in der Bauchhöhle. Archir f. d. gesamte Physiologie. 59. Bd. 1895 .

3) Notk in und Beck, Wiener klin. Wochenschr, 1895.

4) Dubar und Remy, Schmidts Jahrb. 66. Bd.

a) Starling und Tubby, Ebenda. 1895 .

6) Tavel und Lanz, Über die Ätologie der Peritonitis. Mitteilungen aus Kliniken und med. Instituten der Sehweiz. 1. Reihe. Heft 1. 1893.

7) Pawlowsky, Zur Lehre von der Ätiologie, der Entstehungsweise und den Formen der aknten Peritonitis. Virchows Archiv. 117. Bd. 1889.

8) Burginsky, Referat in Baumgartens Jahresbericht über die pathog. Mikroorg. 7. Jahrg. 1891.

9) Wallgreen, Experimentelle Untersuchungen über peritoneale Infektion mit Streptokokken. Zieglers Beitr. 25. Bd. 1899. 
durch diese Resorptionstätigkeit hintangehalten oder vollständig unmöglich werden kann.

Der zweite neben der Resorption einhergehende und vielfach geradezu parallel laufende wichtige Vorgang am Bauchfell, welchem unter pathologischen Erscheinungen eine große Bedeutung zukommt, ist derjenige der Transsudation, beziehungsweise der peritonealen Exsudation. Beide Begriffe sind im Momente des Eintritts pathologischer Prozesse nur schwer oder ïberhaupt nicht mehr auseinanderzuhalten. Die Bedeutung der Transsudation beruht gleichfalls auf der großen Flächenausdehnung der Serosa, welche bekanntlich der Körperoberfläche an Umfang nahezu gleichkommt. Unter physiologischen Verhältnissen - so wird auch heutigentages wohl fast allgemein mit $\mathrm{W}$ eg ner angenommen - befindet sich nur eine kapillare Schicht von freier Flüssigkeit zwischen den einzelnen Flächenabschnitten des Bauchraumes, gerade so viel, daß durch diese Flüssig. keitsmenge die ganze Serosaauskleidung gut befeuchtet wird. Diese Flüssigkeit ist das Produkt des sich zwischen den Serosablättern vollziehenden „Saftstromes" und unterliegt einer fortwährenden Erneuerung, insofern sie beständig neu abgesondert und wieder aufgesogen wird (Wegner). Treffen nun aber irgendwelche Reize das Peritoneum, so besonders Reize mechanischer, chemischer oder thermischer Art, die Reize der Entzündung, oder solche bei Einbringung hypertonischer Lösungen, so erfolgt eine mehr oder weniger ergiebige Vermehrung dieses Flüssigkeitsquantums: Wir finden jetzt ein seröses Ex-oder Transsudat. Bereits W e gner zeigte, daß nach intraabdominaler Injektion von verschiedenen Flüssigkeiten eine Transsudatmenge ausgeschieden werden kann, welche in einer Stunde 4,3 Proz. bis 8,28 P roz. des gesamten Körpergewichtes des Versuchstieres betrug, oder mit anderen Worten: "Wenn nicht durch andere Umstände gewisse Grenzen gesteckt werden, so ist der Organismus imstande, in Zeit von 23-12 Stunden eine Transsudatmenge von seinem Peritoneum aus zu liefern, welche seinem Gesamtkörpergewichtgleichkommt" (S. 106).

Außer diesem Experimentalergebnis, das in unzweideutiger Weise die kolossale Leistungsfähigkeit des Peritoneums in bezug auf Flüssigkeitsabgabe dokumentiert, sind in der Literatur kaum eingehendere Kenntnisse hinsichtlich gerade dieser uns sehr wichtig erscheinenden Eigentümlichkeit des Bauchfells niedergelegt worden. Nur mit dem Produkt dieses Sekretionsvermögens, mit dem Transsudat selbst und mit dem sich normalerweise vollziehenden „Saftstrom" im Peritonealraum hat man sich eingehender befaßt, und hier haben die Untersuchungen dann vor allem darauf 
hingezielt, das Verhalten des Bauchfells in bakteriologischer Beziehung zu erweisen. Gemäl seiner Herkunft durfte man, nachdem durch Traube und Gescheidlen, durch von Fodor, Nuttal, Behring, Nissen, R. Stern, R. Pfeifer usw. die bakterienfeindlichen Eigenschaften des Blutes gegen bestimmte Bakterienarten nachgewiesen waren, schon a priori dieselbe Fähigkeit auch von der peritonealen Transsudationsflüssigkeit erwarten. Den experimentellen Beweis für die Richtigkeit dieser Annahme erbrachten bald $\mathrm{Pan}$ s in i ${ }^{1}$ ), R. Stern ${ }^{2}$ ), Prudden ${ }^{3}$ ) and R. Pfeiffer ${ }^{4}$ ). Wir wissen durch sie, daß das Peritonealsekret für gewisse Bakterien, z. B. für Darmbakterien, eine tötende bezw. entwicklungshemmende Kraft, keine oder wenigstens nur eine ganz geringe für die Eiterkokken, Staphylokokken und Streptokokken, besitzt. In welcher Weise sich aber diese bakterizide Kraft geltend macht und worauf in erster Linie ihre Wirkung auf intraperitoneal eingebrachte Mikroorganismen beruht, ist noch keineswegs mit Sicherheit ergründet. Friedrich ${ }^{5}$ teilt in dieser Frage die Auffassung von Schnitzler und Ewald; nach ihm handelt es sich dabei "nicht um eine spezifische Bakteriolyse (etwa wie sie R. Pfeiffer bei choleraimmunisierten Tieren gegenüber dem Cholerabazillus nachweisen konnte), auch nicht um spezifisch antitoxische Vorgänge im Sinne Behrings, sondern um ganz generell wirksame, individuell schwankende, mehr auf allgemein bakterizide Zellkräfte hinweisende Vorgänge."

Wenn wir nun auch in dem peritonealen Saftstrom und dem Transsudat eine vielleicht nicht unwirksame Schutzvorrichtung zu erblicken haben - und dieses dürfte zum mindesten im Einklang stehen mit dem modernen Wandel in der Auffassung aller entzündlichen Vorgänge -, so bleibt doch der Entscheid offen, inwieweit die Transsudation selbst ein die Bakterizidie fördernder und damit bakterienhemmender Vorgang ist (Friedrich). Es ist ja hinlänglich bekannt, daß in vielen Fällen von akuter Peritonitis der Hyperämie der Serosa ein Flüssigkeitsergul von seiten des Bauchfells auf dem Fuße folgt, ein Erguß, der zunächst meist nur ein reinseröser oder serofibrinöser ist.

Welche pathologische, insbesondere aber klinische Wertschätzung:

1) Pansini, Zieglers Beiträge. 12. Bd. 1893.

2) R. Stern, Zeitschr. f. klin. Med. 15. Bd. 1891.

3) Prudden, zitiert nach Baumgartens Jahresbericht für 1890.

4) R. Pfeiffer, Zeitschr. f. Hygiene und Infektion. 16., 18., 20. Bd.

5) Friedrich, Zur bakteriellen Ätiologie und zur Behandlung der diffusen Peritonitis. Archiv f. klin. Chirurgic. 68. Bd. Heft 2. 
kommt nun diesem Ergub zu? Will das Peritoneum hiermit den Kampf gegen den bakteriellen Feind beginnen und zunächst von diesem Abwehrmittel Gebrauch machen, oder handelt es sich hier einfach um eine pathologisch-anatomisch notwendige, durch die Anwesenheit der Mikroorganismen bedingte Reaktion der Serosa, ähnlich wie sie bei jeder anderen Gewebsinfektion mehr oder weniger auch zustande kommt? Nach theoretischen Erwägungen und nach kliniseher Erfahrung kann man hier wohl eine Antwort vermuten, aber einwandsfrei und begründend kann doch nur das Experiment sprechen.

Auf Anregung und nach dem Entwurf meines Chefs, des Herrn Professor Friedrich, habe ich deshalb den Versuch unternommen, jener Frage durch eine experimentelle Prüfung der Bedeutung des peritonealen Transsudationsstromes nach intraabdominaler Einbringung von Bakterien und Bakterientoxinen näher zu treten. Analoge Versuche in dieser Richtung sind, soweit ich die Literatur überblicke, nicht publiziert worden, wie denn überhaupt die nähere Erforschung: der peritonealen Transsudationsfäbigkeit in den letzten Jabren wesentliche Fortschritte nicht zu verzeichnen hat, obgleich mehr als je der Chirurg mit der Physiologie und Pathologie des Bauchfells praktisch paktiert oder in Konflikt kommt. Man hat sich vielmehr mit der Registrierung des Wegnerschen Befundes begnügt, nicht aber es sich angelegen sein lassen, durch eine Wiederholung oder Nachprüfung seiner Versuche bei anderer Fragestellung und unter neuen Gesichtspunkten einmal ihre Gültigkeit darzutun, sie auszubauen und zu erweitern, und sodann die Bedeutung peritonealer Transsudation auch für pathologische Zustände zu erschließen.

Damit war unsere Versuchsanordnung gegeben: Ehe wir uns mit der Frage nach der Einwirkung von Bakterien und Bakterientoxinen hinsichtlich der Erzeugung einer peritonealen Transsudation befassen konnten, multe zunächst durch Studien über ihre Anregung mittelst anderer Substanzen eine Basis geschaffen werden, nach der jene Einwirkung beurteilt werden konnte; die Wegnerschen Ermittelungen schienen sie nicht detailliert genug zu bieten.

Unsere nachstehend mitgeteilten Versuche sind im Laboratorium der chirurgischen Universitäts-Poliklinik zu Leipzig ausgeführt worden.

Es sei die Bemerkung rorausgeschickt, daß in allen Fällen eine einheitliche Versuchstechnik zur Anwendung kam. Als peritoneale Reizmittel wurden zunächst Flüssigkeiten verwandt. Die mehrfach angefochtene Methode, die Injektion der Flïssigkeiten mittelst der Pravazschen Spritze auszuführen, wurde auch von uns, 
als unkontrollierbar und nicht sicher genug, verworfen. Nach Aufspannen des Tieres, Rasieren und Sterilisieren des Operationsgebietes wurde in der Linea alba, ungefähr in der Mitte zwischen Schwertfortsatz und Nabel eine ca. $1,5 \mathrm{~cm}$ lange Inzision gemacht, dann immer genau die Mittellinie innehaltend das Peritoneum freigelegt und auf Stecknadelkopfweite eröffnet, so daß die Öffnung durch eine nach abwärts eingeführte, stumpfe und etwa $8 \mathrm{~cm}$ lange Injektionskanüle nahezu vollständig ausgefüllt wurde. Um aber möglichst die Garantie zu haben, daß von den injizierten Flüssigkeiten nichts wieder aus der Bauchhöhle abflieben oder ausgepreßt werden konnte, wurde zirkulär um die Kanüle eine Seidennaht gelegt und so lange von einem Assistenten die mit einem chirurgischen Knoten versehenen Fadenenden straff gehalten, bis die Injektion beendet und die Kanüle aus dem Peritonealschlitz herausgezogen war. So gelang es fast regelmäBig, einer Ungenanigkeit der injizierten Flüssigkeitsdosis vorzubeugen und gleichzeitig einen zuverlässigen Sehlub der Peritonealöffnung zu erhalten. Wie sehr auf diesen letzteren Punkt geachtet werden mußte, zeigte uns das unerwünschte Vorkommnis bei einem deshalb ausgeschalteten Versuch, wo wegen einer nicht exakt schließenden Naht durch die transsudierte Abdominalflüssigkeit eine hochgradige Durchtränkung der Bauchdecken in weitem Umkreise von der Injektionsöffnung zustande gekommen war. Schien daher in einigen späteren Fällen die eine Naht nicht ausreichend, so wurde zur Vorsicht noch eine Übernähung ausgeführt. Die kleine Hautwunde wurde dann durch eine fortlaufende Naht geschlossen, ein Gazekollodiumverband gegeben, das Tier vom Versuchsbrett abgespannt und bis zur Beendigung des Versuches in dem geheizten Laboratorium gelassen.

Zur Gewinnung des Transsudates wurde wiederum nach Auf spannen und Reinigen des Tieres dicht unterhalb des ersten Schnittes in der Linea alba eine ca. $6 \mathrm{~cm}$ lange Inzision gemacht, das Peritoneum aber zunächst nur so weit durchtrennt, dab durch den Schlitz eine graduierte, $10 \mathrm{ccm}$ fassende Pipette eingeschoben werden konnte. Erst wenn so ausgiebig wie möglich die freie Bauchhöhlenflüssigkeit zu beiden Seiten der Wirbelsäule ausgehebert war, wurden entsprechend dem Hautsehnitte in der Mittellinie auch die Bauchdecken und das. Peritoneum gespalten, rasch die Intestina auf sterile, warme Kompressen ausgepackt und der Füllungsgrad der Peritoneal- und Mesenterialgefäße festgestellt. Bei der Beurteilung dieses Punktes waren wir stets eingedenk der Wegnerschen Beobachtung, daf nämlich sehr bald nach der Eröffnung der Bauchhöhle die sogenannte Entspannungs- 
hyperämie in die Erscheinung zu treten pflegt. Die Maßnahme des Eventrierens konnte deshalb nicht unterbleiben, weil uns an einer möglichst genauen Bestimmung des Flüssigkeitsquantums gelegen war. Nach der Zurücklagerung der Darmschlingen in die Bauchhöhle wurden sodann die Bauchdecken und die Hautwunde durch eine zweischichtige, fortlaufende Seidennaht geschlossen.

Es mag nochmals erwähnt werden, daß alle operativen Maßnahmen strengst aseptisch ausgeführt wurden. Diesem Umstande verdanken wir es daher, daß wir von 83 Tieren keines an Sepsis oder operativer Peritonitis zugrunde gehen sahen; im Gegenteil konnten wir bei einigen Kaninchen, die mehrere Wochen nach dem ersten Versuch einem zweiten Experiment dienstbar waren, die aseptisch eingebrachte Seidennaht bei absoluter Reaktionslosigkeit der Serosa in den Bauchdecken eingeheilt finden. Auch die Injektionsflüssigkeiten waren in jedem Falle steril; sie wurden stets, auf Körpertemperatur erwärmt, in die Bauchhöhle gebracht.

Dem Beispiel Wegners folgend, suchten auch wir zunächst durch Traubenzuckerlösungen den Transsudationsstrom anzuregen und kennen zu lernen.

Wegner hatte nach dieser Richtung hin drei Versuche ausgeführt. Er injizierte zunächst einem $1922 \mathrm{~g}$ schweren Kaninchen $50 \mathrm{ccm}$ einer konzentrierten Zuckerlösung und fand nach 1 Stunde $215 \mathrm{ccm}$ dünner, klarer, farbloser, leicht eiweißhaltiger Flüssigkeit im Abdomen. Das Tier hatte also 8,28 Proz. (beziehungsweise 8,58 Proz.) seines Körpergewichts an Flüssigkeit abgeschieden. - Bei dem zweiten, $1410 \mathrm{~g}$ schweren Kaninchen trat $1 \frac{1}{2}$ Stunden nach der Injektion von $60 \mathrm{ccm}$ konzentrierter Zuckerlösung unter Krämpfen der Tod ein. Im Abdomen waren $200 \mathrm{ccm}$ leicht rötlich gefärbter, klarer Flüssigkeit enthalten. - In dem dritten Versuch, wo er einem $1840 \mathrm{~g}$ schweren Kaninchen $20 \mathrm{ccm}$ konzentrierter Zuckerlösung injiziert hatte, waren nach einer Stunde $100 \mathrm{ccm}$ eiweißhaltiger, rötlich gefärbter Flüssigkeit in der Bauchhöhle, also $80 \mathrm{cem}$ Transsudat $=4,3$ Proz. des Gesamtkörpergewichtes.

Von der Schluffolgerung Wegners, daß die Transsudation quantitativ sehr wesentlich beeinflußt sei von der Menge der injizierten Flüssigkeit, überzeugten wir uns durch unsere beiden ersten Versuche.

1. Versuch: Einem $1125 \mathrm{~g}$ schweren Kaninchen werden $15 \mathrm{ccm}$ einer konzentrierten Traubenzuckerlösung in die Bauchhöhle gespritzt. Nach einer Stunde waren $88 \mathrm{cem}$ einer klaren, etwas sußlich schmeckenden, ganz schwach blutig gefärbten Flüssigkeit im Abdomen. - Dünndarm etwas kontrabiert, Peritoneum ohne GefäBinjektion. 
2. Versuch: Einem $1250 \mathrm{~g}$ schweren Kaninchen werden $10 \mathrm{ccm}$ einer konzentrierten Traubenzuckerlösung in die Bauchhöhle injiziert. Nach einer Stunde betrug die Flussigkeitsmenge im Abdomen $64 \mathrm{ccm}$. Das Transsudat war ebenfalls klar und kaum rötlich gefärbt. Peritoneum ohne Besouderheiten.

Nach Abzug des injizierten Flüssigkeitsquantums war also in dem einen Falle ein Transsudat von $73 \mathrm{ccm}$, in dem andern von $49 \mathrm{ccm}$ erzeugt. - Geringere Konzentrationsgrade von Traubenzuckerlösung waren nicht imstande, das Peritoneum zur Transsudatabgabe zu veranlassen. Die nachfolgende Tabelle möge dieses illustrieren:

\begin{tabular}{|c|c|c|c|c|}
\hline Nr. & Injiziert wurden & $\begin{array}{l}\text { Dauer der } \\
\text { Einwirkung }\end{array}$ & $\begin{array}{l}\text { Flüssigkeiten im } \\
\text { Abdomen }\end{array}$ & $\begin{array}{c}\text { Resorbiert } \\
\text { also }\end{array}$ \\
\hline $\begin{array}{l}\text { 3. Versuch: } \\
\text { Kaninchen, } \\
3000 \mathrm{~g} \text {. }\end{array}$ & $\begin{array}{c}15 \text { ccm konzentrierte } \\
\text { Zuckerlösung } \\
+15 \mathrm{ccm} \text { Wasser. }\end{array}$ & $\begin{array}{l}2 \text { Stunden } \\
5 \text { Min. }\end{array}$ & $\begin{array}{l}8 \text { cem klarer, schwach } \\
\text { blutiger Flüssigkeit. }\end{array}$ & $22 \mathrm{com}$. \\
\hline $\begin{array}{l}\text { 4. Versuch } \\
\text { Kaninchen, } \\
2530 \mathrm{~g} \text {. }\end{array}$ & $\begin{array}{c}15 \text { cem konzentrierte } \\
\text { Zuckerlösung } \\
+45 \text { ccm Wasser. }\end{array}$ & $\begin{array}{l}2 \text { Stunden } \\
8 \text { Min. }\end{array}$ & $\begin{array}{l}\quad 48 \mathrm{ccm} \text { klarer Flüssig- } \\
\text { keit. }\end{array}$ & $12 \mathrm{ccm}$. \\
\hline $\begin{array}{l}\text { 5. Versueh } \\
\text { Kaninchen, } \\
2250 \mathrm{~g} \text {. }\end{array}$ & $\left\{\begin{array}{c}15 \mathrm{ccm} \text { konzentrierte } \\
\text { Zuckerlösung } \\
-105 \text { ccm Wasser. }\end{array}\right.$ & $\begin{array}{l}2 \text { Stunden } \\
25 \mathrm{Min} .\end{array}$ & $\begin{array}{l}52 \text { cem klarer Flüssig- } \\
\text { keit. }\end{array}$ & $68 \mathrm{cem}$. \\
\hline
\end{tabular}

Ganz ähnlich verhielten sich Kochsalzlösungen intraperitoneal injiziert.

6. Versuch: Einem $1930 \mathrm{~g}$ schweren Kaninchen werden $15 \mathrm{ccm}$ einer 5 proz. Kochsalzlösung in das Abdomen gebracht. Nach 2 Stunden waren nur $2 \mathrm{ccm}$ einer klaren Flüssigkeit aus der Bauchhöhle zu gewinnen.

7. Versuch. Einem Kaninchen von $2000 \mathrm{~g}$ Gewicht werden $15 \mathrm{ccm}$ einer kalt gesättigten Kochsalzlösung injiziert. Nach 20 Minuten setzen klonisch-tonische Krämpfe ein und nach 1 Stunde 20 Minuten erfolgt der Exitus. In der Bauchhöhle waren $68 \mathrm{ccm}$ etwas trübe, nicht blutige Flässigkeit vorhanden. Kéne Hyperämie.

Als gemeinsames Resultat der angeführten Versuche ergibt sich, daß Traubenzucker- und Kochsalzlösungen niederer Konzentration der Resorption anheimfallen, daß dagegen gesättigte Lösungen in der Abdominalhöhle den Transsudationsstrom anzuregen pflegen. Die Menge des Transsudates ist dann direkt proportional dem Quantum der Injektionsflüssigkeit.

Positive Ergebnisse wurden auch durch eine intraabdominale Injektion von Glyzerin gewonnen. Wieder ist es Wegner gewesen, der sich zuerst dieses Mittels zur Erzeugung eines Transsudates bedient hat. In seiner Arbeit teilt er mit, daß er bei einem Kaninchen von $1620 \mathrm{~g}$ Gewicht, dem $25 \mathrm{ccm}$ Glyzerin injiziert war, nach 
$3_{4}$ Stunden ein Transsudat von $105 \mathrm{ccm}$, bei einem $16600 \mathrm{~g}$ schweren Hunde nach $270 \mathrm{cem}$ Glyzerineinspritzung $380 \mathrm{cem}$ Transsudat in 45 Minuten und bei einem $25500 \mathrm{~g}$ schweren Hunde nach 40 Minuten langer Einwirkung von $500 \mathrm{ccm}$ Glyzerin $1300 \mathrm{ccm}$ Transsudat in der Bauchhöhle fand. Weitere Injektionsversuche mit demselben Mittel sind, soweit die Literatur durchgesehen werden konnte, nicht publiziert. Nur Schnitzler und Ewald erwähnen, „daß Glyzerin als Lösungsmittel die (peritoneale) Resorption entschieden verzögert", und erklären diese Tatsache damit, daß "die wechselnd starke, aber recht konstant nachweisbare Flüssigkeitsabsonderung des Peritoneums auf die Einwirkung des Glyzerins hin die Ursache des Verhaltens sei".

Um nun eine genauere Kenntnis der Wirkung des Glyzerins zu erhalten, machten wir gerade mit diesem Mittel zahlreiche Versuche. Es schien uns deshalb zur Injektion in die Peritonealhöhle so besonders geeignet, weil es daselbst nach seinen physiologischen Eigenschaften eine sehr energische Wirkung entfalten mußte, obne doch die Serosa stärker zu alterieren oder gar ernstlich auf die Dauer zu schädigen; sodann deswegen, weil es ja noch heute in der medizinischen Heilwissenschaft, namentlich in der chirurgischen und gynäkologischen Therapie als Lösungsmittel und Vehikel für zahlreiche Medikamente vielfach verwandt wird.

Über seine Eigenschaften, soweit sie für unsere Versuche in Frage kommen, sei Nachstehendes bemerkt: Am bekanntesten dürfte seine hygroskopische, wasserentziehende Eigenschaft sein. Rumpf ${ }^{1}$ ) empfiehlt daher auf Grund seiner Untersuchungen, allen Substanzen, welche eine mehr als oberflächliche Wirkung auf Schleimhäute entfalten sollen, Glyzerin im Prozentverhältnis von 5-10 Proz. zuzusetzen, da es eine wesentliche Beschleunigung der Diffusion bewirke. Eine andere Seite des Glyzerins hat das Tierexperiment ergeben: Die toxische. Nach Husemann ${ }^{2}$, der bereits 1866 die giftige Wirkung des Glyzerins auf Frösche nachgewiesen hatte, beschäftigten sich 10 Jahre später auch Dujardin-Beaumetz und Audigé ${ }^{3}$ ) mit dieser Frage. Durch Versuche an Hunden und Kaninchen zeigten sie, daß chemisch reines Glyzerin in größeren Quantitäten als 0,8 Proz. des Körpergewichtes, subkutan beigebracht, den Tod herbeiführt. Bei den ver-

1) Rumpf, Über Diffusion und Resorption. Deutsche med. Wochenschr. 43. 1900 .

2) Hasemann, Schmidts Jahrb. 184. Bd.

3) Dujardin-Beaumetz und Audigé, Gaz. des Hôpit. 89. 1876. Zitiert nach Schmidts Jahrb. 
gifteten Tieren war Gliederschwäcbe, Hämoglobinurie 1) und Konvulsionen von tetanischem Charakter ${ }^{4}$ ) vorhanden. Pfannenstiel ${ }^{3}$ ) hat anch am Menschen Intoxikationserseheinungen beschrieben, wo behufs Einleitung einer künstlichen Frühgeburt intrauterine Einspritzungen von Glyzerin gemacht waren. Ferner teilt Schellenber g ${ }^{4}$ ) u. a. sogar einen letal verlaufenen Fall von Glyzerinintoxikation nach Jodoformglyzerininjektion aus der Mikuliczsehen Klinik mit, wo bei einem 4 jährigen Kinde nach Auskratzung einer Coxitis tuberculosa die Gelenkhöhlenwunde mit $60-65 \mathrm{~g}$ ausgefüllt war. Sektionsbefund: Nephritis acuta parenchymatosa, Methämoglobininfarkt, Gliaödem, Fettleber.

Daß dem Glyzerin auch eine gewisse bakterizide Kraft innewohnt, hat in neuerer Zeit v. Wunschheim ${ }^{5}$ ) durch Versuche dargetan. Nach ihm ist das unverdünnte käufliche Glyzerin imstande, "auf den Choleravibrio, den Staphylococcus pyogenes aureus, sowie auf Bakterium coli bakterizid einzuwirken." Am längsten halten sich die beiden letztgenannten Bakterienarten in den am meisten Wasser enthaltenden Gemischen.

In unseren Versuchen kam nicht chemisch reines Glyzerin, sondern das offizinelle Präparat, das 28 B ea um esche Grade zeigte, also einen Wassergehalt von etwa 12 Proz. hatte, zur Verwendung.

Wir begannen mit geringen Dosen, injizierten zunächst nur $2 \mathrm{ccm}$ körperwarmen, sterilen Glyzerins und laparotomierten hier, wie auch in den späteren Experimenten, nach verschiedenen Zeitabschnitten, um die Transsudationskraft des Peritoneums zu ermitteln. - Es sei an dieser Stelle die Bemerkung eingefẗgt, daß nur in vereinzelten Fällen die Glyzerininjektion bei den Tieren lebhafteren Widerstand, den sie durch Losreißungsversuche kundgaben, oder Schmerzensäußerung durch Schreien hervorrief; im allgemeinen blieben sie ruhig und ohne merkbare Beeinträchtigung ibres Befindens.

8. Versuch: Um 11 Uhr 30 Minuten vormittags werden einem $1125 \mathrm{~g}$ schweren Kaninchen $2 \mathrm{ccm}$ Glyzerin in die Bauchhöhle eingebracht. Nach halbstündiger Einwirkung sind durch Pipettieren $28 \mathrm{ccm}$ klaren, fast wasserhellen, nicht blatig gefärbten Transsudates aus der wieder er-

1) Filehne, Virchows Archiv. 66. Bd. Heft 2. S. 413. - Luchsinger, Pflïgers Archiv. 11. Bd. S. 502.

2) Langendorf, Eine Glyzerinwirkung. Archiv f. Anat. u. Physiol. Physiol. Teil. 1891.

3) Pfannenstiel, Zentralbl. f. Gynäk. 1894.

4) Schellen berg, Archiv. f. klin. Chirurg. 49. Bd. 1894.

5) v. Wunschbeim, Beeinflußt Glyzerin als Lösungsmittel den Desinfektionswert von Antiseptizis? Archiv f. Hyg, u. Infekt. 39. Bd. 1901. 
öffneten Baucbhöhle zu gewinnen. - Schluß der Bauchwunde durch fortlaufende, zweischichtige Seidennaht. Kollodiumverband. Tier bleibt am Leben.

9. Versuch. Um 9 Uhr 15 Minuten vormittags werden einem kleinen Kaninchen von $1000 \mathrm{~g} 2 \mathrm{ccm}$ Glyzerin in die Bauchhöhle gespritzt. Um 10 Uhr 15 Minuten sind $27 \mathrm{ccm}$ etwas getrübten, nicht blutigen Transsudates vorhanden. Darmgefäße mäßig hyperämisch.

10. Versuch: Um 9 Uhr 52 Minuten werden einem Kaninchen von $1125 \mathrm{~g} 2 \mathrm{ccm}$ Glyzerin intraabdominal injiziert. - Um $12 \mathrm{Uhr} 22$ Minuten enthält die Bauchhöhle $40 \mathrm{ccm}$ fast klares, nicht blutiges Transsudat bei entschieden vorhandener Hyperämie der Mesenterialgefäße.

Wie man sieht, gelingt es schon bei dieser kleinen Glyzerindosis; eine ziemlich beträchtliche Wirkung $\mathrm{zu}$ erzielen. Denn in dem Versuch 8 hat das Tier innerhalb 30 Min. bereits 2,3 Proz. seines Körpergewichtes an Flüssigkeit in die Abdominalhöhle abgeschieden, in dem folgenden betrug das Transsudat nach einer Stunde 2,5 Proz. und im 10. Experiment nach 21/2 Stunden 3,4 Proz. des Körpergewichtes.

Da eine toxische Wirkung des Glyzerins sich bei diesen untermittelgroßen Tieren in keiner Weise geltend gemacht hatte, glaubten wir bei kräftigen, ausgewachsenen Kaninchen unbeschadet das doppelte Quantum einbringen zu dürfen.

11. und 12. Versuch: In einem kräftigen Kaninchen von $3000 \mathrm{~g}$, bezw. $2500 \mathrm{~g}$ Gewicht werden $4 \mathrm{ccm}$ Glyzerin in das Abdomen injiziert. In beiden Fällen können nach genau 2 Stunden nur $5 \mathrm{ccm}$ einer nicht völlig klaren Flüssigkeit aus der Bauchhöhle entnommen werden. Keine Hyperämie.

13. Versuch, Intraperitoneale Injektion von $4 \mathrm{ccm}$ Glyzerin bei einem großen Kaninchen. Nach 4 Stunden sind $2 \mathrm{ccm}$ einer blutigserösen Flüssigkeit im Abdomen.

14. Versuch: Desgleichen bei einem großen Kaninchen. Nach 8 Stunden sind ca. $2 \mathrm{ccm}$ einer trüben, blutig gefärbten Flüssigkeit im Abdomen. Mäßige Hyperämie der Mesenterialgefäße.

15. Versuch: Desgleichen. Nach 25 Stunden ist nur $1 \mathrm{ccm}$ einer wenig getrubten Flüssigkeit im Abdomen. Peritoneum glatt und glänzend.

Die vorstehende Versuchsreihe wurde einmal aus der Erwägung heraus angestellt, daß man bei dieser Glyzerindosis eine Vermehrung des Transsudates erwarten durfte; sodann sollte ermittelt werden, nach welcher Zeit dasselbe wieder vom Peritoneum durch Resorption fortgeschafft werden könnte. Durch die analogen Versuche 11 und 12 sollte zunächst die Menge des erwarteten Transsudates festgestellt werden, um danach die Resultate der übrigen 3 Experimente beurteilen zu können. Überraschend ist der negative Ausfall. Es ist wohl möglich, daß bei der raschen Wirkung des Glyzerins die Flüssigkeitsabsonderung in kurzer Zeit sistierte und nach 2 Stunden der 
Erguß bereits wieder der Resorption anheim fiel; aber dann kann das Transsudat doch nur eine reeht minimales gewesen sein, da für die Aufsaugung eines größeren Quantums dieser immerhin doch kurze Zeitraum nicht ansgereicht bätte. Der eigentliche Grund liegt jedoch wohl darin, daß wir uns hier sehr kräftiger und widerstandsfähiger Tiere bedienten. Wenigstens zeigen die drei folgenden Kontrollversuche, dal von der Größe des Tieres der Ausfall des Versuches wesentlich beeinflufit wird.

16. Versuch: Einem Kaninchen von $19 \mathrm{~g}$ Gewicht werden $4 \mathrm{cem}$ Glyzerin in die Abdominalhöble injiziert. - Nach einer Stunde beträgt die Transsudatmenge $35 \mathrm{cem}$; es ist klar, schwach blutig gefärbt. Keine Hyperämie. - Zweischichtige Bauchnaht. Tier lebt.

17. Versuch: Einem Kaninchen von $1125 \mathrm{~g}$ Gewicht werden $4 \mathrm{ccm}$ Glyzerin intraabdominal injiziert um 11 Uhr 15 Minuten vormittags. Nach 2 Stunden sind $42 \mathrm{cem}$ einer klaren, nicht blutig gefärbten Flüssigkeit vom Peritoneum ausgeschieden. Ganz mäßige Hyperämie der Darmgefäße. Banehfell ohne Besonderes. Temperatur vor der Operation um 11 Uhr 11 Minuten vormittags $38,8^{\circ}$, um 1 Uhr $36,3^{\circ}$. Abends Tier munter, Temperatur $38^{\circ}$.

18. Versu $\mathrm{eh}$ : Einem Kaninchen von $1125 \mathrm{~g}$ Gewicht werden $4 \mathrm{cem}$ Glyzerin intraperitoneal injiziert.

Nach $31 / 2$ Stunden sind $65 \mathrm{ccm}$ sanguinolenter, mäßig getrubter Flussigkeit in der Bauehhöhle vorhanden. Bei der Injektion entleerten sich aus der stecknadelkopfgroßen Peritonealöffinung mehrere Kubikzentimeter einer bräunlich-roten Flüssigkeit. Tier wird zur Transsudatgewinnung getötet: am Magen finden sich Coceidien 1).

Im Gegensatz zu den analogen Versuchen 11-15 ist bei den zuletzt angeführten jedesmal der Transsudationsstrom angeregt worden; die gefundenen Transsudatmengen entsprechen etwa 1,68 Proz, 3,4 Proz. und 5,3 Proz. des Körpergewichtes jedes Tieres. Das Glyzerin hat auch in diesen Fällen noch keine toxische Nebenwirkung hervorgebracht, da kein Tier eine wahrnehmbare Beeinträchtigung seines Wohlbefindens zeigte, obgleich in dem einen Versuche eine beträchtliche Temperaturabnahme (um 2,5 Grad) festgestellt wurde.

1) Um möglichst genau die Anwesenheit einer freien Abdominallüssigkeit zu eruieren, drängten wir in allen Fällen den vorliegenden Darmteil, meist das Coecum, mit der eingeführten stumpfen Kanüle vor der Injektion beiseite; dann wurde durch Zusammenpressen der Bauchdecken des Tieres mittelst flach aufgelegter Hände von den Seiten nach der Mediane zu mehrfach versucht, dic event. vorhandene Bauchhöhlenflüssigkeit zu entfernen. Nur in dem 18. Versuch und ferner in einem nicht referierten, flossen dabei mehrere Knbikzentimeter derselben ab; sonst in einigen Fällen bis höchstens tă Tropfen. Wir dürfen daher annehmen, daß eine freie Flüssigkeit im Abdomen der Tiere nicht vorhanden bezw. daf sic zu geringfügig war, um bei einer genauen Feststellung des Transsudates ernstlich in Rechnung zu kommen. 
19. Versuch: Intraperitoneale Injektion von $6 \mathrm{ccm}$ Glyzerin bei einem $1875 \mathrm{~g}$ schweren Kaninchen um $9 \mathrm{Uhr} 3$ Minuten vormittags. Um 12 Uhr 30 Minuten nachmittags, also $31 / 2$ Stunden nach der Einspritzung, sind aus der Bauchhöhle $57 \mathrm{~cm}$ eines klaren, bämorrhagisehen Transsudates zu entfernen. Temperatur vor der Operation 38,80; um $10 \mathrm{Uhr} 20$ Minuten vormittags $355^{\circ}$ und um 12 Uhr 35 Minuten nachmittags 36,10 .

Hier ist neben einer Vermehrung des Transsudates die Temperaturabnahme noch bedeutender, wie in dem 17. Versuche. Da aber auch diese Glyzerindosis sonst ron dem mittelgroßen Kaninchen unbeschadet vertragen wurde, steigerten wir dieselbe in einer größeren Versuchsreihe um weitere $2 \mathrm{ccm}$.

20. Versu c h: Um 9 Uhr 15 Minuten vormittags werden einem $1750 \mathrm{~g}$ schweren Kaninchen $8 \mathrm{ccm}$ Glyzerin in die Abdominalhöhle injiziert. Um 10 Uhr 45 Minuten sind $91 \mathrm{ccm}$ eines klaren, dunnflassigen, nicht blutig aussehenden Transsudates vorhanden. Peritoneum ohne Besonderes, keine Hyperämie. Mikroskopisch sind im Transsudat nur sehr wenig rote und ganz vereinzelt auch weiße Blutkörperchen nachzuweisen.

21. Versuch: Um 9 Uhr 20 Minuten vormittags werden einem großen Kaninchen von $1600 \mathrm{~g}$ Gewicht $8 \mathrm{ccm}$ Glyzerin injiziert.

Bald nach der Injektion Tier entschieden leidend, ab und zu stellt sich kurzdauernder Opisthotonus ein. Exitus um 12 Uhr 45 Minuten vormittags. In der Bauchhöhle sind $72 \mathrm{ccm}$ klaren Transsudates, das beim Stehen in Zimmertemperatur zum größten Teile gallertig gerinnt; mikroskopisch ist derselbe Befund wie in 20 zu erheben.

22. Versuch: Um 8 Uhr 50 Minuten vormittags werden einem untermittelgroßen Kaninchen von $1375 \mathrm{~g}$ Gewicht $8 \mathrm{ccm}$ Glyzerin in die Bauchhöhle gebrach h

Um 12 Uhr 30 Min. nachmittags ist im Abdomen eine Flussigkeitsmenge von $64 \mathrm{ccm}$ enthalten. Transsudat klar, nicht sanguinolent. Etwa 9 Uhr 35 Minuten vormittags bekommt das Tier Opisthotonus und klonischtonische Krämpfe der Extremitäten, die jedesmal etwa 30 Sekunden anhalten. Die Sektion des am folgenden Tage verstorbenen Tieres ergibt keine Besonderheiten, Peritoneum glatt, glänzend, ohne Gefäßinjektion.

23. Versuch: Um 8 Uhr 40 Min. vormittags intraabdominale Einspritzung von $8 \mathrm{ccm}$ Glyzerin bei einem $2000 \mathrm{~g}$ schweren Kaninchen. Nach fast vier Stunden werden $70 \mathrm{ccm}$ eines klaren, nicht blutig tingierten Transsudates gewonnen.

24. Versuch: Um 8 Uhr 50 Min. vormittags werden einem Kaninchen von $2000 \mathrm{~g}$ Gewicht $8 \mathrm{~cm}$ injiziert.

Um 4 Uhr 5 Minuten nachmittags findet sich ein tribes, mit kleinen Flocken durchsetztes, mäßig blutig aussehendes Transsudat von $60 \mathrm{~cm}$ im Abdomen des Tieres. Peritoneum ohne Besonderes.

25. Versuch: Um 8 Uhr 40 Min. nachmittags werden einem $1500 \mathrm{~g}$ schweren Kaninchen $8 \mathrm{ccm}$ in die Bauchhöhle gebracht.

Nachmittags um 3 Uhr 30 Min. können $50 \mathrm{ccm}$ eines leicht getrübten Transsudates aufgehebert werden. 
26. Versuch: Um 9 Uhr 28 Min. werden einem $2000 \mathrm{~g}$ schweren Kaninchen $8 \mathrm{ccm}$ Glyzerin injiziert. Nach 9 Stunden sind in der Bauch höhle nur $5 \mathrm{ccm}$ einer etwas trüben Flüssigkeit vorhanden. - Peritoneum in mäßigem Grade hyperämisch, etwas mehr die Mesenterialgefäße.

27. Versuch: Um 9 Uhr vormittags intraperitoneale Injektion von $8 \mathrm{ccm}$ Glyzerin bei einem $2000 \mathrm{~g}$ schweren Kaninchen.

Nach 10 Stunden finden sich $25 \mathrm{ccm}$ einer mäßig getrtbten, nicht blutigen Flïssigkeit im Abdomen vor. - Peritoneum glatt, glänzend, mit beträchtlicher Hyperämie, besonders der Mesenterialgefäße.

28. Versuch: Um 11 Uhr vormittags intraperitoneale Injektion von $8 \mathrm{ccm}$ Glyzerin bei einem Kaninchen von $2000 \mathrm{~g}$ Gewicht. - Abends $8 \mathrm{Uhr}$ werden $8 \mathrm{cem}$ einer trtben Flüssigkeit, die beim Stehen alsbald gerinnt, pipettiert. Bauchfell glatt, spiegelnd, kaum hyperämisch.

29. Versuch: Um 9 Uhr vormittags intraperitoneale Injektion von $8 \mathrm{ccm}$ Glyzerin bei einem Kaninchen von $2000 \mathrm{~g}$ Gewieht.

Nach 251/2 Stunden sind in der Bauchböhle nur $3 \mathrm{ccm}$ einer klaren Flüssigkeit enthalten; Peritoneum ohne Besonderes.

Was zunächst die Menge des Transsudates anbelangt, so zeigen diese Versuche mit $8 \mathrm{~cm}$ Glyzerin stets ein positives Resultat, wenn einige Stunden, bis etwa 7, nach der Injektion die Ausheberung vorgenommen worden ist; die Flüssigkeitsausscheidung betrug bis 5,14 Proz. des Körpergewichtes. Sodann sehen wir aus dieser Reihe, dab das Maximum des Transsudates nach ein bis zwei Stunden erreicht ist und dab ferner der Ergub danach wieder einer schnellen Resorption anheimfällt; $\mathrm{nach} 10$ Stunden ist er entweder schon ganz aufgesaugt oder nur noch zum kleineren Teile vorhanden, je nach der Größe und dem Kräftezustand des Versuchstieres. Das Peritoneum selbst hat in allen Fällen ein unverändertes Aussehen geboten, nur im 26. und 28. Versuche ist ein mäBiger, im 27. ein stärkerer Grad von Hyperämie zu konstatieren gewesen, ein Befund, der jedoch zu einer begründeten Sehlußfolgerung nicht berechtigen dürfte 1 ).

1) Interessant sind in dieser Beziehung die Ergebnisse von Buchner (Mïnchner med. Wochenschr. 1899. Heft 39 u. 40). Er injizierte kleine Mengen (1 cem) von 60-, 70-, 80 prozent. oder von absolutem Alkohol in die Bauchböhle von Meerschweinchen. Nach 5-6 Stunden fand sich in der Regel ein seroshämorrhagisches Exsudat und rosafarbene Injektion der Serosa. Die untere Seite des Magens zeigte subseröse Blutaustritte, der Dünndarm eine dunkelblaurote-rosa Verfärbung. Bei absolntem Alkohol waren diese Erscheinungen am stärksten ausgeprägt; sehr auffallend war die starke Injektion der Gefäße, namentlich der Mesenterialgefäße. Bei Methylalkohol waren sie weniger vorhanden, bei weitem am bedeutendsten beim Propylalkohol, "1 ccm Propylalkohol intraperitoneal bewirkt beim Meerschweinchen innerhalb 6 Stunden intensive blaurote Verfärbung des ganzen Darmtraktus bis herunter zum Rektum, inkl. 
In zwei Fällen tritt zum ersten Male eine ausgesprochene toxische Einwirkung in die Erscheinung (21. und 22. Versuch), die sich durch klonisch-tonische Krämpfe und durch Opisthotonus kundgegeben hat. Dujardin-Beaumetz und Ardigé (1.c.) erklären diese Wirkung. des Glyzerins durch eine Schädigung der Nervenzentra, namentlich des Rückenmarkes, analog der Wirkung der Alkohole, zu denen ja das Glyzerin als dreiwertiger Vertreter der Gruppe gehört, und auch Langendorff (l. c.) führt sie auf eine „Reizung des Nervensystems durch die Wasserentziehung" zurück.

Daß hierbei die Aufnahme des Glyzerins in den Kreislauf wohl die wichtigste Rolle spielt, zeigen die Untersuchungen des Glyzeringehaltes in verschiedenen Transsudaten, die ich der großen Freundlichkeit der Herren Professor Siegfried und Privatdozent Dr. Burian 1) vom hiesigen physiologischen Institute verdanke. Von ihnen wurde das überraschende und interessante Ergebnis ermittelt, daß in den $91 \mathrm{cem}$ des vom 20 . Versuch stammenden Transsudates nur $0,4163 \mathrm{~g}{ }^{\prime}(=0,3293 \mathrm{ccm})$ Glyzerin und in den $72 \mathrm{ccm}$ des 21 . Versuches nur $0,3259 \mathrm{~g}(=2784 \mathrm{ccm})$ Glyzerin enthalten war; es muß also in beiden Fällen über $7,5 \mathrm{ccm}$ Glyzerin im Blute zirkuliert haben!

Die Beschaffenheit des Transsudates zeigte im allgemeinen keine wesentliche Unterschiede; es war von klarem oder etwas trübem Aussehen, dünnflüssig, von blasser oder etwas rötlicher Farbe (vergl. Wegner, S. 88 und 89), je nach dem Gehalt an roten Blntkörperchen, die sich mikroskopisch stets nachweisen ließen. Meist bildete sich sehr bald nach der Ausheberung durch Fibrinausscheidung eine lockere, manchmal auch eine etwas festere Gerinnung, nur selten blieb das Transsudat ganz flüssig. Zwecks bak teriologis cher Verwertung wurde es gleich aus der Bauchhöhle, jedesmal $\mathrm{zu} 10 \mathrm{ccm}$, in sterile Reagenzröhren pipettiert und im Wasserbade von 39 Grad auf Eigentemperatur gehalten. Sodann wurden auf je zwei Röhren Abimpfungen von virulentem, im bängenden Tropfen Eigenbewegungen zeigendem Bakterium coli und von Staphylococcus pyogenes aureus vorgenommen, während die übrigbleibenden Röhren desselben Transsudates zur Kontrolle unbeschickt blieben; zur Verwertung in dieser Weise gelangte von der vorstehenden Reihe das Transsudat von Versuch 22-27,

Magen, mit massenhaften subserösen Blutergüssen, außerdem sehr starkes hämorrhagisches Traussudat; kurzum die Wirkung auf die Gefäße ist eine ganz außerordentlich starke".

1) Den genannten Herren erlaube ich mir, auch an dieser Stelle für ihre Nühewaltung meinen verbindlichsten Dank auszusprechen. 
ferner von Versuch 8, 17 und von 34, 38 und 39. Nach 24, beziehungsweise 48 stündigem Verweilen im Thermostaten war in den meisten mit Coli beschickten Röhren eine intensive Gasentwicklung zustande gekommen, sie fehlte nur in den Röhren des Transsudates 27 und 25. In allen Fällen jedoch konnten durch Untersuchung im hängenden Tropfen Stäbchenbakterien in mehr oder weniger lebhafter Eigenbewegung nachgewiesen werden. Im mikroskopischen Methylenblaupräparat zeigten sie ein sehr variables Aussehen: neben dem Bakterium coli yon gewöhnlicher Beschaffenheit war anffallend eine Reihe von Stäbchenbakterien, die den Eindruck von Involutionsformen machten.

Hiernach scheint dem Transsudat eine, wenigstens stark bakterizide Kraft nicht inne zu wohnen, denn in jedem Falle hatten sich die Bakterien im Transsudat weiterentwickelt, allerdings nicht in sotypischen Formen und nicht so reichlich wie auf Agar oder in Bouillon.

Die in die Transsudatröhren gebrachten Stap hy lokok ken zeigten ebenfalls regelmäßig Wachstum; nur waren hier in bezug auf Quantität der Kokken grölsere Unterschiede vorhanden. Zahlreihe, einzeln und auch in Träubehen liegende Kokken waren in Deckglaspräparaten aus den Transsudaten 8,17 und 22, weniger aus denen der übrigen Versuche zu finden.

Wir kommen zum

30. Versuch: Einem $1100 \mathrm{~g}$ schweren Kaninchen werden $10 \mathrm{ccm}$ Glyzerin in die Abdominalhöhle injiziert. Unter kurzandauernden Zuckungen der Extremitäten tritt $21 / 2$ Stunden post op. der Exitus eill. $70 \mathrm{cem}$ eines klaren, etwas blutig tingierten Transsudates im Abdomen, Peritoneum ohne Besonderes, keine Hyperämie.

31. Versuch: Um 1 Uhr 36 Min. nachmittags werden einem Kaninchen von $2050 \mathrm{~g}$ Gewicht $10 \mathrm{ccm}$ Glyzerin intraperitoneal injiziert. Gegen 3 Uhr setzen bei sehr frequenter Atmnng klonisch-tonische Krämpfe, verbunden mit Nackenstarre ein, die im allgemeinen 15-25 Sekunden dauern und anf dem Höhestadium nach $1-2-3$ Minuten Pause wiederkehren. Gegen Abend klingen die Zuckungen ziemlich schnell ab, am nächsten Morgen zeigt das Tier ein kaum noch beeinträchtigtes Allgemeinbefinden und am Nachmittage hat es sich völlig wieder erholt.

32. Versuch: Um 1 Uhr 43 Minuten nachmittags werden einem kleinen, sehr munteren Hunde von $3000 \mathrm{~g}$ Gewicht $12 \mathrm{ccm}$ Glyzerin in das Abdomen injiziert. Im Verlauf des Nachmittags zeigt sich der Hund matt, abgespannt, hat jedoch keine Krämpfe. Abends ist er bereits munterer und am nächsten Morgen von der früheren Lebendigkeit.

33 . Versuch: Um 8 Uhr 30 Minuten vormittags werden einem kräftigen Hund von $8000 \mathrm{~g}$ Gewieht $32 \mathrm{cem}$ Glyzerin injiziert. Nachmittags ist er weniger mobil, abends bereits munterer. Keine Krämpfe. Am folgenden Morgen ist das Tier munter wie früher. 
34. Versuch: Um 9 Uhr 11 Minuten vormittags intraperitoneale Injektion von $44 \mathrm{ccm}$ Glyzerin bei einem $6250 \mathrm{~g}$ schweren Pintscher. Nach $121 / 2$ Stunden werden in Äthernarkose $160 \mathrm{ccm}$ eines schwach blutig anssehenden Traussudates aus der Bauchhöhle entfernt. Keine Hyperämie der Mesenterialgefäße, Peritoneum glatt glänzend. Zweischichtige Bauchnaht, Kollodiumverband, Hund lebt.

Aus diesen Versuchen resultiert, daß nach Injektion von $10 \mathrm{ccm}$ Glyzerin beim Kaninchen stets schwere toxische Wirkungen eintreten, denen nur ein sehr kräftiges Tier zu widerstehen vermag. $10 \mathrm{ccm}$ dürten demnach als äußerste Maximaldosis angesehen werden, aber auch nur für ganz kräftige und widerstandsfähige Kaninchen, für Tiere unter $1800 \mathrm{~g}$ Gewicht etwa müssen schon $8 \mathrm{ccm}$ als das höchst zulässige Maß bezeichnet werden. Die Hunde vertragen die Injektion weit besser, auch wenn ihnen die für die Kaninchen gefundene Maximaldosis, auf ihr Körpergewicht umgerechnet injiziert, ist.

Das Transsudat beträgt im 30 . Versuch 3,45 Proz. des Körpergewichtes, eine in Anbetracht der für dies Tier letalen Dosis im Vergleich zu früher gefundenen Werten geringe Menge.

Um nun die Grenze der Transsudationsfähigkeit des Peritoneums zu ermitteln, sind in den folgenden Versuchen große Glyzerindosen injiziert worden.

35. Versuch: Um 11 Ohr 6 Minuten nachmittags werden einem kräftigen Kaninchen von $2200 \mathrm{~g}$ Gewicht $22 \mathrm{ccm}$ Glyzerin in die $\mathrm{Ab}$ dominalhöhle gespritzt.

Nach 1 Stunde 51 Minuten tritt der Exitus letalis ein, ohne daß irgend welehe Krampferscheinungen ausgelöst waren. Im Abdomen finden sich $198 \mathrm{ccm}$ eines klaren, stark blutig gefärbten Transsudates. Peritoneum glatt und spiegelnd, unbedeutende Injektion der Mesenterialgefäße.

36. Versuch: Um 1 Uhr 20 Min. nachmittags werden einem $2150 \mathrm{~g}$ schweren Kaninehen $40 \mathrm{ccm}$ Glyzerin intraperitoneal injiziert.

Nach 11/2 Stunden Exitus letalis. Zwei Stunden darauf wird die Sektion vorgenommen: im Abdomen $166 \mathrm{ccm}$ eines klaren, stark blutig gefärbten Transsudates. Peritoneum ohne Besonderes, kaum bemerkbare Hyperämie der Mesenterialgefäße.

37. Versuch: Um 9 Uhr 35 Minuten nachmittags werden einem kräftigen Zughund von $2000 \mathrm{~g}$ Gewicht $266 \mathrm{ccm}$ Glyzerin injiziert.

Nach 21/4 Stunde tritt der Exitus ein. Im Abdomen finden sich $1300 \mathrm{~cm}$ eines blutigen, klaren Transsudates. Peritoneum ohne Befund, keine Hyperämie.

Die ermittelten Transsudatmengen entsprechen für den 35 . Versuch 8 Proz. des Körpergewichtes, für den 36. rund 6 Proz. und für den letzten 5,17 Proz., Resultate, die mit den $\$$ Wegnerschen Beobachtungen genau übereinstimmen. In diesen Fällen hochgradiger Glyzerinwirkung sind gegen früher ungleich mehr rote Blutkörperchen mit 
ausgeschieden worden, so daß der Erguß jedesmal einen stark hämorrhagischen Charakter angenommen hat. Ganz außerordentlich ist in dem 37. Beispiel die Resorption des Glyzerins gewesen, wie von Professor Siegfried und Dr. Burian nachgewiesen wurde: in $110 \mathrm{ccm}$ der blutig serösen Flüssigkeit fanden sie nur $1,3777 \mathrm{~g}$ (=1,0899 cem) Glyzerin, so dab also nahezu $250 \mathrm{ccm}$ Gly zerin in der kurzen Zeit von $75 \mathrm{Min}$. in den Körperkreislauf übergegangen sein müssen.

Durch frühere Versuche war festgestellt, daß bei einer Injektion von $8 \mathrm{ccm}$ Glyzerin nach 3 Stunden ein Transsudat von etwa $70 \mathrm{ccm}$ in der Abdominalhöhle zu erwarten ist. Es entstand nun die Frage, ob man nicht diese Menge durch subkutane Flüssigkeitszufuhr in irgendeiner Weise zu beeinflussen imstande ist. A priori sollte man vermuten, daß dadurch eine Verm ehrung des Transsudates eintreten müßste, zumal wo man durch Indienststellung einer ausgedehnten subkutanen Resorptionsfläche dem Organismus schnell ein großes Quantum von Flïssigkeit zuzuführen 1) und so die Folgen einer Wasserverarmung des Körpers wirksam zu coupieren vermag.

Die zu diesem Zwecke angestellten Versuche sind technisch so ausgeführt worden, daß zunächst das Glyzerin in die Bauchhöhle gebracht und dann so schnell wie möglich an 6-8 Stellen des Bauches und Rückens bei langsamer Entleerung der $12 \mathrm{cem}$ fassenden Spritze eine körperwarme, physiologische Kochsalzlösung injiziert wurde. Um einem Wärmeverlust des Tieres vorzubeugen, wurde es gleich danach bei Zimmertemperatur in Decken eingepackt.

38. Versuch: Um 9 Uhr 30 Minuten vormittags werden einem $1750 \mathrm{~g}$ schweren Kaninchen $8 \mathrm{~cm}$ Glyzerin intraperitoneal injiziert. Gleich nach Schluß der Bauchnaht subkutane Injektion von $120 \mathrm{ccm}$ physiologischer Kochsalzlösung. Temperatur vor der Operation $39^{\circ}$; um $1 / 212: 38^{0}$.

Um 12 Uhr 30 Min. können aus der Bauchhöhle $47 \mathrm{ccm}$ eines klaren, ziemlich blutig gefärbten Transsudates ausgebebert werden. Peritoneum ohne Besonderes. Keine Hyperämie.

39. Versuch: Um 10 Uhr vormittags werden einem $1875 \mathrm{~g}$ schweren Kaninelen $8 \mathrm{ccm}$ Glyzerin injiziert ins Abdomen.

Sodann: subkutane Injektion von $120 \mathrm{cem}$ physiol. Kochsalzlösung.

Temperatur vor der Operation: $38,7^{\circ}$, um $3 / 112 \mathrm{Uhr}$ vormittags $34^{\circ}$. Gegen 11 Uhr vormittags bekommt das Tier einen mittelstarken Krampfanfall, der etwa 30 Sekunden dauerte.

Um 1 Uhr nachmittags sind in der Banchhöhle $58 \mathrm{ccm}$ einer klaren, ziemlich sanguinolenten Flitssigkeit vorhanden.

1) Vgl. Klapp, S. 262. 
Es scheint hiernach, als ob die subkutane Injektion eine Verminderung, keinesfalls aber eine Vermehrung der Transsudatmenge zur Folge gehabt hat. Auffallend ist auch das hämorrhagische Aussehen des Ergusses, und der bedeutende Temperaturabfall, der trotz der genannten Vorsichtsmabregel eingetreten war.

Bei größeren Glyzerindosen blieb ebenfalls die durch eine gleichzeitige subkutane Wasserzufuhr erwartete Wirkung auf den Umfang der Transsudatbildung aus.

40. Versuch: Einem kräftigen Kaninchen von $2875 \mathrm{~g}$ Gewicht werden $15 \mathrm{ccm}$ Glyzerin intraperitoneal injiziert und gleichzeitig subkutan $160 \mathrm{ccm}$ NaCl-Lösung. Nach 1 Stunde $2 \mathrm{Min}$. tritt unter langsamem Erlöschen der Körperfunktionen der Exitus ein.

In der Bauchhöhle $90 \mathrm{ccm}$ klares, sanguinolentes Transsudat. Peritoneum ohne Besonderes.

41. Versueh: Einem kräftigen Kaninchen von $2125 \mathrm{~g}$ Gewicht werden $15 \mathrm{~cm}$ Glyzerin und gleichzeitig $210 \mathrm{~cm} \mathrm{NaCl-Lösung} \mathrm{subkutan}$ injiziert.

Nach 1 Stunde 20 Min. Exitus, wie bei 40 . Im Abdomen $80 \mathrm{ccm}$ klaren, weniger blutig aussehenden Transsudates. Peritoneum ohne Besonderes. Keine Hyperämie.

Wir hofften, durch jenes die zu erwartende Transsudatmenge weit übersteigende Maß von subkutaner Flüssigkeitszufuhr trotz der letalen Glyzerindosis die Tiere am Leben erhalten zu können; daß es nicht gelang, wird dem Zusammenwirken mehrerer Faktoren zuzuschreiben sein: Der Schwere des operativen Eingriffes an sich, der Glyzerinwirkung, die sich diesmal jedoch nicht durch Krampfauslösung dokumentierte, dem unvermeidlichen Wärmeverlust und der gesteigerten Arbeitsleistung des Organismus, einmal zu transsudieren und sodann die subkutanen Wassermengen zu resorbieren. Wie bestrebt der Organismus gewesen, dieser letzten Anforderung zu entsprechen, davon überzeugten wir uns gleich nach dem Exitus durch Inzisionen an den Infiltrationsstellen: Es war deutlich wahrzunehmen, daB ein beträchtlicher Teil bereits durch Resorption fortgeschafft war. Dennoch war eine Vermebrung der Transsudatmenge ausgeblieben.

Durch einige weiterte Versuche sollte ermittelt werden, ob k or pus$\mathrm{k}$ uläre Elemente als solehe in der Bauchböhle einen Transsudatstrom hervorzurufen imstande seien. - W e g n er hatte seinen Versuchstieren Farbstoffpartikelehen (Tusche, Zinnober, Kohlenpulver) in das Abdomen gebracht und gefunden, daf diese Stoffe ohne weiteres vom Zwerchfell aufgesogen werden. Auch Schnitzler uud Ewald brachten feste Körper in die Bauchhöhle von Kaninchen, 0,1 g metallisches 
Jod, 0,2 und 0,5 g Jodkali und Ferrocyankalium. Die genannten Substanzen wurden relativ rasch in der Peritonealflüssigkeit gelöst und waren alsbald im Harn nachweisbar. Ähnlich fielen ihre Versuche mit halbfesten Substanzen aus (Gelatine- und Agarzylinder, die mit Formalindämpfen vorbehandelt und mit 2proz. Jodkali imprägniert waren). "Nachdem $0,1 \mathrm{~g}$ Jod in etwa 45 Stunden in der Bauchhöhle gelöst und ausgeschieden war, $0,1 \mathrm{~g}$ Jod aber mehr als $200 \mathrm{~g}$ Wasser zur Lösung braucht, können wir uns eine beiläufige Vorstellung von der Intensität des zwischen den Peritonealblättern ablaufenden Saftstromes machen" (l. c. S. 359.) Schnitzler und Ewald hatten sodann einige Messerspitzen von sterilem Streusand und ferner Ruß (von einer Terpentinflamme) der Bauchhöhle von Kaninchen einverleibt und festgestellt, daß dadurch die Resorption eine Verzögerung. nicht erleidet; auch 5 sterile Glaskugeln und eine apfelgroße Zelluloidkugel äußerten intraperitoneal keinen Einfluß auf die resorptiven Vorgänge.

Wie man sieht, beschäftigen sich die referierten Versuche mit der Frage, ob die Resorption im peritonealen Raum durch Einbringung von festen Substanzen tangiert wird. Der negative Ausfall derselben läßt schon von vornherein die Vermutung zu, daß auch die Transsudation dadurch nicht angeregt wird. Die Richtigkeit dieser Annahme wird durch unsere Versuche bestätigt.

42. Versuch: Einem $3000 \mathrm{~g}$ schweren Kaninchen werden 4 sterile, etwa kirschgroße Wattebäuschchen durch eine ca. $1 \mathrm{~cm}$ lange Peritonealöffnung nach verschiedenen Richtungen in die Bauchhöhle geschoben.

Nach 3 Stunden ist im Abdomen keine freie Fllissigkeit nachzuweisen; nur haben sich die Wattebäuschchen, von denen zwei tief $z$ wischen Dünndarmschlingen lagen, mit Flüssigkeit vollgesogen. Keine Hyperämie.

43. Versuch: Einem $2100 \mathrm{~g}$ schweren Kaninchen werden durch einen $0,5 \mathrm{~cm}$ langen Peritonealschlitz 3 gehäufte Messerspitzen sterilen Streusandes mittelst eines nach Angabe von Professor Friedrio h gefertigten Trichters an verschiedenen Stellen (zwischen Dünndarmschlingen) einverleibt.

Nach 3 Stunden $1,5 \mathrm{ccm}$ einer klaren, serösen Flüssigkeit im Abdomen. Der Sand findet sich zu kleinen Klümpchen geballt an den betreffenden Stellen vor, irgendwelche Reizerscheinungen sind nicht vorhanden.

44. Versuch: Einem $2500 \mathrm{~g}$ schweren Kaninchen werden in gleicher Weise 7 gehäufte Messerspitzen sterilen Lykopodiums in die Bauchhöhle gebracht.

Nach 3 Stunden ist etwa $1 \mathrm{ccm}$ klarer Flüssigkeit im Abdomen vorhanden. Keine Hyperämie, keine Reizerscheinungen.

45. Versuch: Einem $1900 \mathrm{~g}$ schweren Kaninchen werden 2 gehäufte Messerspitzen im Mörser verriebenen Pfeffers in das Abdomen gebracht. 
Nach $3^{3} / 4$ Stunden $4 \mathrm{ccm}$ einer klaren serösen Fliissigkeit im $\mathbf{A b}$. domen. Keine Reizersebeinungen.

Der durch zweistundige Heißlufteinwirkung sterilisierte Pfeffer hatte bedeutend an Schärfe verloren, doch war der Pfeffergeschmack noch nachhaltig anf der Zunge zu spüren.

Überblicken wir nun zunächst die durch die bisher mitgeteilten Versuche gewonnenen Resultate, so können wir sie kurz in folgende Sätze zusammenfassen:

Traubenzucker und Kochsalzlösungen in hoben Konzentrationen regen das Peritoneum zur Flüssigkeitsabgabe an; von den Lösungen niederer Konzentration dagegen wird nach kurzer Zeit ein großer Teil resorbiert gefunden, nicht aber der Transsudationsstrom angeregt.

Glyzerininjektionen in das Abdomen pflegen fast stets eine Absonderung seröser peritonealer Flüssigkeit z ur Folge zu haben, deren Menge proportional der Menge der injizierten Dosis ist. Während kräftige Tiere auf 2 bezw. 4 ccm nicht zu reagieren brauchen, ist bei kleineren die durch diese Dosen hervorgerufene Transsudatmenge ziemlich beträchtlich. Werden $6 \mathrm{ccm}$ injiziert, so wird auch bei kräftigen Tieren ein ergiebiger Transsudationsstrom angeregt. $10 \mathrm{ccm}$ stellen im allgemeinen das höchst zulässige Injektionsmaß dar; bei noch höheren Dosen tritt meist unter klonisch-tonischen Krämpfen der Exitus ein, die Menge der freien Abdominalflüssigkeit ist dann am beträchtlichsten und kann bis 8 Proz. des Gesa m tkörpergewichtes betragen.

Je nach der Glyzerindosis ist auch der Gehalt an Blutkörperchen im Transsudat ein verschiedener : bei hohen Injektionsquanten ist er sehr groß, so daß der Erguß hämorrhagisch aussieht, bei kleinen ist er gering oder nicht nachweisbar.

Das Glyzerin geht sehr schnell und in großen Mengen in den Blutkreislauf über, wo es als toxische Wirkung Krämpfe klonisch-tonischen Charakters und Opisthotonus auslösen kann; das Transsudat enthält nur noch ganz geringe Quantitäten der injizierten Gabe.

In bakteriologischer Beziehung zeigt sich das Transsudat als nicht bakterizid für Bakterium coli und Staphylococcus pyogenes aureus; das Bakterium coli entwickelt sich in demselben fort, meist bei lebhafter Gasbildung und fast stets unter Erhaltung der Eigenbewegung und Änderung seiner Formen. Nicht immer gleich- 
mäBig in quantitativer Beziehung gedeihen im Transsudat Staphylokokken.

Eine Vermehrung der Transsudatmenge läßt sich, auch bei hoher Glyzerindosis, durch gleichzeitige subkutane Injektion von physiologischer Kochsalzlösung an mehreren Stellen nicht erzielen; ferner kann dadurch bei letaler Glyzerindosis der Exitus nicht verhindert werden.

Sterile korpuskuläre Elemente sind, sofern sie das Bauchfell weder mechanisch schädigen noch chemisch reizend beeinflussen, nicht imstande, eine Flüssigkeitsabsonderung zu bewirken.

Nach diesen Ermittelungen, durch welche, wie ersichtlich, die Wegnerschen Resultate ihre Bestätigung und Erweiterung erfahren, sollte zunächst durch einige weitere Versuche festgestellt werden, $o b$ das Peritoneum auf eine intraabdominale Injektion von Bakteri enreinkulturen mit einer unmittelbaren Flüssigkeitsabsonderung zu antworten pflegt oder nicht.

Im strengen Sinne des Wortes dïrfen wir jetzt zwar nicht mehr von einer Transsudation reden, da diese ja ohne entzündliche Reizvorgänge meist auf mechanischem Wege zu entstehen pflegt, hier jedoch mehr oder weniger eine entzündlich peritonitische Affektion im Spiele ist. Doch sei es gestattet, diesen Begriff beizubehalten, bezw. ihn der Exsudation gleichzusetzen, zumal, wo wir ja nur die ersten Anfänge der Flüssigkeitsabsonderung von seiten des Peritoneums im Auge haben, die zunächst meist auch seröser oder sero-fibrinöser Natur ist und sich qualitativ im wesentlichen dann nur durch höheren Eiweifigebalt von dem einfachen Transsudat unterscheidet.

Eine Durchsicht der Versuchsprotokolle von Autoren, die eine experimentelle Peritonitis hervorrufen konnten (Pawlowsky, Wallgreen [1. c.]), ergibt, daß des öfteren eine größere oder geringere Menge eines serös-hämorrhagischen ("katarrhalischen") Exsudates im Abdomen der Tiere, wenn der Tod bald erfolgt war, enthalten gewesen ist. Genaue Angaben aber, ob sich als direkte Folge der bakteriellen Injektion, ähnlich wie bei der Erzeugung einer chemischen Peritonitis, die Flüssigkeitsabsonderung einstellt, sind nicht vorhanden, und nur ans klinischer Erfahrung weiß man, daß sich bei akuter Peritonitis in der Regel bald an die entzündliche Hyperämie der Serosa eine fibrinöse und seröse Exsudation anschließt, von denen die letztere bei wechselnder Quantität je nach der Ausdehnung ein Flüssigkeitsquantum bis zu einigen Litern, gelegentlich bis zu 20, 
liefern kann. Da nun aber andererseits kleinere seröse Ergüsse schnell wieder resorbiert werden können, so dürfen wir für unsere Versuche, wenn überhaupt, nur in den ersten Stunden nach der Injektion einen solchen peritonealen Ergub erwarten.

Injiziert haben wir unseren Versuchstieren selbstgezüchtete Reinkulturen von Staphylococeus pyogenes aureus, dessen pathogenetische Bedeutung für diee Peritonitis des Menschen, wenn auch nur für einzelne Fälle, gesichert ist, und ferner Reinkulturen von Bakterium coli, nächst den Streptokokken der häufigste Erreger jener Erkrankung $\left.{ }^{1}\right)$.

46. Versuch: Einem $2350 \mathrm{~g}$ sehweren Kaninchen wird eine Aufschwemmung von Staphylokokkenkulturen, die in 3 Agarröhren 38 Stunden gewachsen waren, in $10 \mathrm{ccm}$ physiologischer Kochsalzlösung intraabdominal injiziert.

Nach 3 Stunden 20 Min. sind in der Bauchhöhle ea. 2 eem einer etwas triben, sanguinolenten Flüssigkeit vorhanden, die unter dem Mikroskop zahlreiche zellige Bestandteile (Leukozyten) und nur ganz vereinzelt Kokken aufweist. Peritoneum glatt, glänzend, in geringem Maße hyperämiseh.

47. Versuch: Einem $1900 \mathrm{~g}$ schweren Kaninchen werden injiziert $8 \mathrm{ecm}$ Staphylokokkenbouillonkultur von 36 Stunden. Das Allgemeinbefinden an dem nächsten Tage kaum noch beeinträchtigt. Tier bleibt am Leben.

Einem Meerschweinchen, dem $3 \mathrm{ccm}$ derselben Kultur mit Pravazscher Spritze injiziert wurde, erliegt nach etwa 14 Stunden; im Abdomen $5 \mathrm{ccm}$ einer truben, sanguinolenten Flüssigkeit. Peritoneum stark hyperämisch, etwas verwaschen aussehend. Abimpfungen aus Bauchhöhle ergeben reichliches Wachstum von Staphylokokken in Reinkultur.

48. Versuch: Einem $3400 \mathrm{~g}$ schweren Kaninchen wird eine 4 Tage alte Colibouillonkultur von $12 \mathrm{ccm}$ in das Abdomen injiziert. Spontaner Exitus nach 1 Stunde 50 Min. ea. $17 \mathrm{com}$ einer klaren, nicht sanguinolenten Flissigkeit im Abdomen. Peritonenm ohne Injektion.

49. Versuch: Einem Kaninchen von 1800 Gewicht wurden $8 \mathrm{ccm}$ derselben Colibouillon injiziert. Nach $2^{1 / 4}$ Stunden plötzlicher Exitus. Das Tier, das ruhig dagesessen hatte, bekam einen starken Krampfanfall, streckte alle Extremitäten von sich und starb nach wenigen Atemzügen. In der Bauchhöhle ca. $6 \mathrm{ccm}$ etwas trüber Flïssigkeit. Keine Hyperämie des Peritonenms. Abimpfungen aus Banchhöhle und rechtem Herzen ergeben Colibazillen in Reinkultur.

50. Versuch: Einem $1800 \mathrm{~g}$ schweren Kaninchen werden $15 \mathrm{ccm}$ derselben Colibouillon injiziert. Tier nach 3 Stunden getötet; im $\mathrm{Ab}$ domen $18 \mathrm{ccm}$ einer hellen, leicht getrubten Flüssigkeit.

50. Versuch: Einem $2100 \mathrm{~g}$ schweren Kaninchen werden die in 3 Agarröhren durch 38 Stunden gewachsenen, in $10 \mathrm{ccm}$ Kochsalzlösung aufgeschwemmten Colikulturen injiziert.

1) Vgl. No thnagel, Die Erkrankungen des Darms und des Peritoneums. 1898 
Nach 3 Stunden ea. $5 \mathrm{ccm}$ einer triben, sanguinolenten Flussigkeit im Abdomen. Peritoneum von mäBiger Hyperämie, glatt, glänzend.

52. Versuch: Einem $1300 \mathrm{~g}$ schweren Kaninchen werden $20 \mathrm{ccm}$ einer zweitägigen Boullonkultur injiziert. Das Tier zeigt während der Daner der Einwirkung keine Störung in seinem Befinden. Nach 3 Stunden sind $21 \mathrm{ccm}$ einer hellen, nicht blutigen Flüssigkeit in der Bauchhöhle. Peritoneum anämisch, glatt.

53. Versuch: Einem $1500 \mathrm{~g}$ schweren Kaninchen werden $6 \mathrm{ccm}$ einer 40 stiundigen Colibouillonkultur injiziert. Spontaner Tod nach 23 Stunden. Im Abdomen $1 \mathrm{cem}$ klare Flussigkeit, Därme und Bauchfell anämisch, ohne Auflagerungen; Dickdarm stark gashaltig, ebenso der ganze Dündarm mit schäumigem, dünnflüssigem Inhalt erfullt. Abimpfungen aus Banchhöhle, Leber und rechtem Herzen ergeben Colibazillen in Reinkultur. Bemerkenswert ist ferner, daß bald nach der Injektion Diarrhöen aufgetreten sind.

54. Versuch: Einem $2000 \mathrm{~g}$ schweren Kaninchen werden $10 \mathrm{ccm}$ einer 36 stündigen Colibouillonkultur injiziert. Am nächsten Tage setzen starke Diarrhöen ein, die einen ublen Geruch verbreiten; sie halten in unvermindertem Maße bis zum Exitus nach $4_{4}^{3 / 4}$ Tagen an. Sektion: ca. $3 \mathrm{ccm}$ einer truben Flussigkeit im Abdomen, Peritoneum glatt und glänzend, ohne Auflagerungen, nicht hyperämisch.

Weder durch eine Injektion von Staphylokokken noch von Bakterium coli in Reinkulturen ist das Peritoneum zur Flüssigkeitsabgabe angeregt oder gereizt worden. Wenn die Bakterien mit der indifferenten physiologischen Kochsalzlösung zusammen in das Abdomen eingebracht sind, so findet sich der größere Teil derselben na ch drei Stunden ohne merkbaren Schaden für das Tier aufgesogen; in der restierenden Bauchhöhlenflüssigkeit sind dann mikroskopisch und kulturell nur ganz versehwindend wenige Kokken oder Kolistäbchen nachzuweisen. DaB aber die injizierten Mikroorganismen virulent waren, ist für Versuch 46 und 47 , wo demselben Stamm entnommene Staphylokokken verwandt wurden, aus dem Kontrollexperiment mit dem Meerschweinchen zu ersehen, das bereits nach 14 Stunden einer beginnenden Peritonitis erlegen ist; und fur den Versuch 51 zeigt es in analoger Weise das Resultat der 53. Beobachtung.

In den Fällen, wo Colibouillon eingespritzt war, waren nach drei Stunden (Versuch 50 und 52) nur 3 bezw. 1 cem Flüssigkeit mehr im Abdomen zu finden, als die injizierte Menge betrug; die gefundene Menge sah heller aus, als die Bouillon und war nicht sanguinolent. Ein ganz unbedentendes Plus von freier Abdominalfliussigkeit mit demselben Charakter war auch im 48. Experiment zu konstatieren. Dieser Befund fällt deshalb etwas auf, weil das Tier der Injektion rasch erlag und trotzdem jenes Flüssigkeitsquantum gefunden wurde. Hier könnte man an eine Abgabe ron Flïssigkeit durch das Peritoneum 
denken, zumal in 49. Versuch die injizierte Colibouillon nach nur $25 \mathrm{Min}$. längerem Verweilen in der Bauchhöhle quantitativ sich vermindert und dann den überraschend plötzlichen Tod zur Folge gehabt hatte.

Auffallend ist auch das Ergebnis der beiden letztgenannten Ver. suche; beide Male traten starke Diar rh öen auf, durch die das 54. Versuchstier an Erschöpfung zugrunde ging.

Die nähere Untersuchung der im 51. Versuch nach drei Stunden gewonnenen Bauchhöhlenflüssigkeit ergab im hängenden Tropfen die Anwesenheit von zahlreichen, teils nicht mobilen, teils in Eigenbewegung befindlichen Stäbchenbakterien, die im gefärbten Präparat ähnlich wie die im Transsudat gezüchteten (s. o.) ein mannigfaltiges Aussehen erkennen ließen: neben dem normalen Bakterium coli waren meistens dickere und plumpere Stäbchen anzutreffen, die mit Methylenblau vielfach intensiv gefärbt, fast stets allein lagen.

Genauere Untersuchungen über das Verhalten des Bakterium coli machte Radziewsky 1), der seinen Versuchstieren (Meerschweinchen) tödliche Dosen injizierte und in jeder Phase bis zum Exitus die Peritonealflüssigkeit mikroskopierte. Er fand, daß einerseits eine Vermehrung, andererseits eine Deformation und Auflösung der Bakterien in der Bauchhöhle erfolgt. Bereits 15 Minuten nach der Injektion büßte eine Anzahl von Mikroorganismen teilweise oder völlig ihr Tinktionsvermögen ein, während nach einer Stunde die größere Mehrzahl sich intensiv mit Methylenblau-K $\ddot{u}$ hn $\mathrm{e}$ färbte.

Da in unseren zuletzt mitgeteilten Versuchen dieinjizierten Bakterienreinkulturen in keinem Falle einen nennenswerten Erguß zur Folge gehabt hatten, sondern durch eine mehr oder weniger rasche Resorption aus der Bauchhöhle fortgeschafft waren, wollten wir fernerhin ihre Einwirkung auf das Peritoneum nach Anregung bezw. auf der IÏhe des Transsudationsstromes einer Prüfung unterziehen. Zur Erzeugung des Transsudates wurde das uns aus den früheren Experimenten gut bekannte Glyzerin genommen.

Die Versuche, zu denen besonders kräftige Tiere ausgewählt wurden, wurden technisch in der Weise ausgeführt, dab die stumpfe Kanüle nach ihrer eingangs beschriebenen Einführung und nach vollendeter Glyzerininjektion verschlossen wurde und in situ blieb, um so auch der späteren Einbringung der Kulturen dienen zu können; erst danach wurde sie behutsam entfernt und das Tier vom Versuchsbrett abgespannt. Auf diese Weise konnte eine zweite Laparotomie

1) Radziewsky, Beitrag zur Kenntnis der Bakterium coli. Zeitschr. f. Hygiene. 34. Bd. 1900. 
vermieden werden, ohne daß die Versuchstechnik an Exaktheit eingebült hätte.

55. Versuch: Um 9 Uhr 12 Minnten vormittags werden einem $1600 \mathrm{~g}$ schweren Kaninchen $6 \mathrm{ccm}$ Glyzerin injiziert und um $10 \mathrm{Uhr}$ 12 Minuten vormittags $6 \mathrm{ccm}$ einer 40 stindigen Colibouillonkultur.

Temperatur um 9 Uhr $39^{\circ}$; um $10 \mathrm{Uhr} 50 \mathrm{Min} .33,3^{\circ}$; um $12 \mathrm{Uhr}$ $35,9^{\circ}$. Um 12 Uhr $52 \mathrm{Min}$., also nach 2 Stunden $40 \mathrm{Min}$. sind aus dem Abdomen $62 \mathrm{ccm}$ einer klaren, ziemlich dickflüssigen, kaum hämorrhagischen Fltissigkeit zu pipettieren. Abimpfungen von Banch, Leber, Niere und Herz auf Agaragar ergeben Reinkulturen von Bakt. coli,

56. Versuch: Um 9 Uhr 20 Min. vormittags werden einem $1875 \mathrm{~g}$ sehweren Kaninchen $6 \mathrm{ecm}$ Glyzerin und um $10 \mathrm{Uhr} 20 \mathrm{Min}$. vormittags ebenfalls $6 \mathrm{ccm}$ derselben Colibouillon injiziert.

Temperatur vor der Operation $38,9^{\circ}$; um 10 Uhr 52 Min. 33,7 um $7 \mathrm{Uhr} 30 \mathrm{Min}$. nachmittags $34,5^{\circ}$. Kurz nach $8 \mathrm{Uhr}$ nachmittags, also nach etwa 11 Stunden, tritt der Exitus ein. Sektion: kein Tropfen freier Flüssigkeit im Abdomen, Banchfell kanm hyperämisch, etwa rosafarben, mit etwas verwaschener Zeichnung und ganz feinen, punktförmigen Auflagerungen. Darmschlingen nicht verklebt. Abimpfungen aus Bauch, Leber und Herz sind positiv.

57. Versuch: Um $9 \mathrm{Uhr}$ vormittags werden einem $2000 \mathrm{~g}$ schweren Kaninchen $8 \mathrm{~cm}$ Glyzerin und um $10 \mathrm{Uhr} 6 \mathrm{ccm}$ einer viertägigen Colibouillon injiziert.

Temperatur vor der Operation $39,5^{\circ}$, um $11 \mathrm{Uhr} 30 \mathrm{Min}$. vormittags $35,3^{0}$. Um $12 \mathrm{Ubr} 35 \mathrm{Min} .$, also etwa $3^{1 / 2}$ Stunden post. inject. Glyz., enthält das Abdomen $84 \mathrm{cem}$ einer schwach blutig gefärbten, leicht getrübten Flussigkeit. Das Peritoneum bietet keine Besonderheiten, keine Hyperämie.

58. Versuch: Um 9 Uhr 10 Min. vormittags werden einem $2100 \mathrm{~g}$ schweren Kaninchen $8 \mathrm{ccm}$ Glyzerin und um $10 \mathrm{Uhr} 10 \mathrm{Min}$. vormittags $6 \mathrm{ccm}$ derselben Colibouillon injiziert.

Temperatur vor der Operation 39,40, um 11 Ohr 35 Min. vormittags $35,4^{\circ}$, um 5 Uhr 15 Min. nachmittags $35^{\circ}$ und um 7 Uhr 10 Min. nachmittags $34,8^{\circ}$. Gegen 1/28 Uhr nachmittags Exitus. Sektion: im Abdomen $18 \mathrm{ccm}$ eines trüben, mäßig sanguinolenten, dickflussigen Ergusses. Das parietale Blatt des Bauchfells etwas getrübt, weniger das viszerale; einzelne Darmschlingen zeigen ganz lockere Verklebungen. Abimpfungen aus Bauch, Leber und rechtem Herzen ergeben nach 24 stundigem Wachstum kolossale Mengen von Bakterium coli.

Als gemeinsames Resultat dieser Versuche finden wir, dab eine Vermehrung der Transsudation durch die Bakterieninjektion nicht erzielt worden ist. Der negative Ausfall befremdet desbalb nicht, weil ja auch bei alleiniger Einverleibung reiner Bakterienkulturen das Peritoneum in dieser Hinsicht unbeeinflubt geblieben ist.

Im übrigen stimmen die gefundenen FIüssigkeitsmengen quantitativ mit unseren bisherigen Ergebnissen 
genau überein; auch der starke Temperaturabfall in diesen vier Fällen als Folge der Transsudatabscheidung ist uns aus mehreren früheren Versuchen bekannt.

Nach einer anderen Richtung hin aber scheinen diese Beobachtungen bemerkenswert. Denn während in keinem Falle, wo nur reine Kolikulturen injiziert waren, irgendwelche peritonitische Symptome aufgetreten sind, sind im 56 . und 58. Experiment deutlich Zeichen einer beginnenden Entzündung von seiten des Bauchfells vorhanden gewesen. - Wie ist nun diese Beobachtungstatsache zu erklären? Schon Wegner (l. c.) hat auf die Bedeutung einer Stagnation zersetzungsfähiger Flüssigkeit in der Bauchhöhle als einen für die Entstehung einer Peritonitis wichtigen Faktor aufmerksam gemacht und anch Grawitz ${ }^{1}$ pflichtet dieser Ansicht bei. Nach seiner Überzeugung kommt dann eine eitrige Peritonitis zustande, wenn pyogene Mikroorganismen in eine Abdominalhöhle gebracht werden, welche eine stagnierende Nährflüssigkeit enthält oder deren Serosa durch ätzende Substanzen geschädigt worden ist. Auch in der Anwesenheit eines Ascites wird, wie Reichel²) hervorhebt, ein prädisponierendes Moment für eine septische Peritonitis nach Eröffnung der Bauchhöhle gesehen. „Das gesunde Peritoneum wird Herr über die bei strenger Antisepsis ja nur in geringer Menge mit ihm in Berührung kommenden Spaltpilze, ein abnormer Inhalt der Bauchböhle, Ascites, gewährt ihnen Zeit zur massenhaften Entwicklung und zur Entfaltung ihrer furchtbaren Kräfte."

In unserem 56. und 58. Versuche war nun eine größere Quantität freier Abdominalflüssigkeit enthalten, in der, wie festgestellt, sich Bakterium coli weiter entwickelt und die ferner frühestens erst nach 10 Stunden durch Resorption fortgeschafft sein konnte.

So waren hier, wenn auch die Zeit kurz erscheint, die Bedingungen gegeben, unter denen sich peritonitische Erscheinungen einstellen konnten.

Daß es sich in unseren Fällen nicht um eine sogenannte chemische Peritonitis handelte, wie sie z. B. Pawlowsky dureh Trypsin experimentell hervorgebracht und wie sie Tavel und Lanz mehrere Male durch Bakteriengifte zustande kommen sahen, zeigte einmal das indifferente Verhalten des Glyzerins der peritonealen

1) Grawitz, Statistischer und experimentell pathologiseher Beitrag zur Kenntnis der Peritonitis. Charitê-Annalen. XI. 1886.

2) Reichel, Beiträge zur Ätiologie und chirurgischen Therapie der septischen Peritonitis. Deutsche Zeitschr. f. Chirurgie. 30. Bd. 1890. 
Experim. Beitrüge zur Kenntnis des Transsudationsvorganges am Eauchfell. 449

Endothelschicht gegenüber in den früheren Versuchen und sodann enthielten die injizierten Colibouillonkulturen doch zu geringe Mengen solcher Toxine. Auch unsere folgenden Beobachtungen sprechen dagegen, wo den Tieren sehr bedeutende Mengen von Bakterienfiltraten und -sterilisaten intraabdominal beigebracht wurden und bei keinem eine chemische Peritonitis entstand.

59. Versuch: Einem $1500 \mathrm{~g}$ sehweren Kaninchen werden $10 \mathrm{ccm}$ Colifiltrat I) injiziert. Nach $2 \frac{1}{2}$ Stunden sind $3-4 \mathrm{ccm}$ trüber Flüssigkeit im Abdomen. Peritoneum anämisch, Abimpfungen ans Bauchhöhle negativ.

60. Versuch: Einem $12 \mathrm{~g}$ schweren Kaninchen werden $10 \mathrm{ccm}$ Colifiltrat injiziert. Temperatur in den nächsten Tagen normal $(38,9$ bis $39,50)$. Bald nach der Injektion stellen sich ibelriechende Diarrhöen ein, denen das Tier am 14. Tage erliegt. Sektion ohne Befund.

61 . Versach: Einem $2250 \mathrm{~g}$ schweren Kaninchen werden $20 \mathrm{cem}$ Colifiltrat injiziert. Nach $21 / 2$ Stunden sind $38 \mathrm{cem}$ etwas trüber, gelblicher Flüssigkeit im Abdomen. Peritoneum obne Besonderes.

62. Versuch: Einem $2000 \mathrm{~g}$ sehweren Kaninchen werden $20 \mathrm{ccm}$ Colifiltrat injiziert. Am 6. Tage Exitus. Das Tier hat am 1. und 2. Tage post. op. keine auffälligen Erscheinungen geboten, von da ab verschlechtert sich sein Befinden. Sektion: Abimpfungen aus Bauchhöhle negativ, Bauchfell glatt und glänzend, ohne Auflagerungen, nicht hyperämisch. Darmschlingen nicht verklebt, Dünndarm kontrahiert. Keine freie Flüssigkeit im Abdomen.

63. Versuch: Einem $1300 \mathrm{~g}$ sehweren Kaninchen werden $20 \mathrm{ccm}$ Staphylokokkenfiltrat injiziert. Nach 21/2 Stunden sind noch $2 \mathrm{ecm}$ ziemlich klarer Flussigkeit in der Bauchhöhle. Anämie der Darmserosa, Bauchfell glatt und spiegelnd.

64. Versuch: Einem $1300 \mathrm{~g}$ schweren Kaninchen werden $20 \mathrm{ccm}$ Stapbylokokkenfiltrat nach Prof. Friedriehs Technik injiziert. Tier bietet keine Besonderheiten in seinem Befinden, bleibt am Leben.

65 . Versuch: Einem $1300 \mathrm{~g}$ schweren Kaninchen werden $20 \mathrm{cem}$ Colisterilisat injiziert. Nach 3 Stunden sind im Abdomen $17 \mathrm{ccm}$ einer trüben, etwas flockigen Flüssigkeit. Keine Hyperämie des Peritoneums.

1) Herr Dr. Heller, Assistenzarzt an der chirurgischen Universitäts-Poliklinik, batte die Freundlichkeit, mir dic in dieser Versuchsreihe genannten Filtrate und Sterilisate darustellen und zu überlassen.

Die zur Herstellang dionenden Kultaren waren bei $37,5^{\circ} \mathrm{C}$. in Fleischwasserpeptonkochsalaboullon gezüchtet, die Kolikulturen 4, bezw. 10 Trge und die Staphylokokken 9 Tage lang. Die Filtrate wurden mit Chamberland-Filter nol Luftpumpe hergestellt, durch Abimpfnngen auf ihre Keimfreiheit geprüft und bis zum Gebrauch im Eisschrank aufbewahrt. Die Sterilis ate wurden durch 10 Stunden langes Erwälmen auf $60^{\circ} \mathrm{C}$. in Wasserbade gewonnen. Durch $A b$ impfungen von Stunde zu Stunde wurde die Lebensfathigkeit der Bakterien geprïft. Nit der 6. Stunde blieben die Abimpfungen in der Regel schon ohne Wachstum. 
66. Versu c b: Einem $2400 \mathrm{~g}$ sehweren Kaninchen werden $20 \mathrm{ccm}$ Colisterilisat injiziert. Nach 6 Stunden ist keine freie Flüssigkeit im Abdomen mehr vorhanden. Peritoneum ohne Besonderes.

67. Versuch: Einem $1600 \mathrm{~g}$ schweren Kaninchen werden $20 \mathrm{ccm}$ Staphylokokkensterilitat injiziert. Nach 3 Stunden finden sich $20 \mathrm{cem}$ etwas getrubter Flussigkeit in der Banchböhle. Peritoneum ohne Hyperämie, glatt.

68. Versuch: Einem $1250 \mathrm{~g}$ schweren Kaninehen werden $20 \mathrm{ecm}$ Staphylokokkensterilisat injiziert. Nach 6 Stunden sind im Abdomen $11 \mathrm{ccm}$ einer truben, etwas flockigen Flüssigkeit. Banchfell ohne Besonderes.

Außer im 61. Versuche konnte, wie wir seben, in keinem Falle eine Vermehrnng der Bauchhöblenflüssigkeit nachgewiesen werden; man darf daher wohl schlieben, dabim allgemeinen weder durch Bakterienfiltrate noch -sterilisate als solche auf das normale Peritoneum unmittelbar ein transsudationsbefördernder Reiz ausgeübt zu werden pflegt. Die injizierten Fliissigkeiten wurden vielmehr resorbiert, ohne daß sich eine toxische Wirkung bemerkbar gemacht hatte.

Diese negativen Ergebnisse stehen nicht im Einklang mit den Pawlowskyschen Resultaten. Pawlowsky (l. c. S. 486) gelang es, durch Injektion von $30 \mathrm{ccm}$ Filtrat einer 8 tägigen, ferner von $20 \mathrm{ccm}$ und $15 \mathrm{ccm}$ einer 4 tägigen Bouillonkultur von Staphylococcus aureus bei fünf Meerschweinchen jedesmal eine Peritonitis haemorrhagica exsudativa hervorzurufen, der die Tiere am nächsten Tage bereits erlegen waren. Bei der Sektion wurde dann außer einer großen Quantität serös-hämorrhagischen Exsudates, intensive Injektion der Serosa der Gedärme und des Peritoneums gefunden. - Wie diese Differenzen zu erklären sind, ob sie aus der Verschiedenheit der Versuchstiere, der injizierten Menge und der Toxinvirulenz oder der Versuchstechnik resultieren, mag dahin gestellt bleiben. Auch nach 6 stündiger intraabdominaler Einwirkung müßte in unseren Fällen eine peritonitische Reizung sich bereits gezeigt haben, falls die Kürze der Zeit angeschuldigt werden sollte. Überdies zeigten ja auch unsere Kontrolltiere keinerlei Reaktion auf die Injektion hin.

Auch bei bestehendem Erguß, der auf dieselbe Weise, wie bei Versuch 55-58 durch Glyzerin erzeugt wurde, folgte einer Filtratoder Sterilisatinjektion keine Vermehrung peritonealer Flüssigkeitsabsonderung.

69. Versuch: Um 9 Uhr 50 Min. vormittags werden einem $2250 \mathrm{~g}$ schweren Kaninehen $6 \mathrm{ccm}$ Glyzerin und um $10 \mathrm{Uhr} 50 \mathrm{Min}$. vormittags $10 \mathrm{ecm}$ Colifiltrat injiziert. Um $1 \mathrm{Uhr} 20 \mathrm{Min}$. nachmittags sind $57 \mathrm{cem}$ fast klarer, etwas sanguinolenter Flüssigkeit im Abdomen. Keine Hyperämie.

70. Versuch: Um $10 \mathrm{Uhr}$ vormittags werden einem 1625 schweren Kaninchen $6 \mathrm{ccm}$ Glyzerin und um 11 Uhr 7 Min. vormittags $10 \mathrm{ccm}$ Coli- 
Experim. Beiträge zur Kenntnis des Transsudationsvorganges am Bauchfell. 451

filtrat injiziert. Nach $51 / 2$ Tagen Exitus letalis. 2 ccm Flïssigkeit im Abdomen. Peritoneum ohne Besonderes. Am Tage nach der Operation bekommt das Tier anhaltende Diarrhöen.

71. Versuch: Um 10 Uhr vormittags werden einem $1300 \mathrm{~g}$ sehweren Kaninchen $6 \mathrm{ccm}$ Glyzerin und um 11 Uhr $10 \mathrm{ccm}$ Staphylokokkonfiltrat injiziert. Um 1 Uhr $15 \mathrm{Min}$. nachmittags sind $66 \mathrm{ccm}$ einer dünnflüssigen, fast klaren, etwas blutigen Flüsigkeit im Abdomen. Peritoneum ohne Besonderes, keine Hyperämie.

72. Versuch: Um $10 \mathrm{Uhr} 15 \mathrm{Min}$. vormittags werden einem $1200 \mathrm{~g}$ schweren Kaninehen $6 \mathrm{ccm}$ Glyzerin and um 11 Uhr $15 \mathrm{Min}$. vormittags $10 \mathrm{ccm}$ Staphylokokkensterilisat injiziert. Um 12 Uhr $15 \mathrm{Min}$. nachmittags sind $69 \mathrm{cem}$ etwas blutiger, mäßig truber Flissigkeit im Abdomen. Peritoneum glatt und glänzend, nicht hyperämisch.

Ein zusammenfassender Rückblick auf die letzten 36 Versuche zeigt uns, dab die Einbringung von Staphylococcus pyogenes aureus und Bakterium coli in Reinkulturen in eine normale Bauchhöhle im allgemeinen keinen Flüssigkeitsergub hervorbringt. Sind die Mikroorganismen mit einer indifferenten Flüssigkeit zusammen eingespritzt, so findet vielmehr bald eine Resorption des injizierten Materials statt; langsamer geht dieselbe von statten, wenn die Kulturen mit Bouillon dem Abdomen einverleibt werden.

Peritonitische Erscheinungen sind dadurch nicht zur Entwicklung gelangt; geht das Versuchstier zugrunde, so ist die plötzliche und massenhafte Uberschwemmung der Blutbahn durch die Mikroorganismen als Todesursache anzuschuldigen. Für einen Fall kommt ferner eine "toxische Diarrhöe" in Betracht, die auch in einigen andern Beobachtungen sich gezeigt hat.

Nach Anregung des Transsudationsstromes zeigt sich eine Bakterieninjektion ebenfalls auBer stande, weiter transsudationsbefördernd einzuwirken; dagegen treten schon nach verhältnismäbig kurzer Zeit deutlich Erscheinungen einer beginnenden Peritonitis auf, die den Exitus letalis zur Folge haben.

Bakterienfiltrate und sterilisate von Staphylococeus pyogenesaureus und Bakterium coli haben weder für sich allein, noch bei künstlich erzeugtem Transsudat eine Einwirkung auf das Peritoneum auszuäben vermocht; sie sind im allgemeinen, ohne das Versuchstier zu alterieren, resorbiert worden, und nur in einem Falle könnte das Auftreten einer "toxischen" Diarrhöe auf die Einwirkung von Colifiltrat zurückgeführt werden. 
Zum SchluB sei es mir gestattet, meinem hochgeehrten Chef, Herrn Professor Friedrich, für die Anregung zu dieser Arbeit, für die fördernde Unterstützung bei Ausführung derselben und für die gütige Gewährung aller Hilfsmittel auch an dieser Stelle meinen aufrichtigsten, verbindlichsten Dank auszusprechen.

Desgleichen möehte ich dem Volontärassistenten an der chirurgischen Universitäts-Poliklinik, Hern Dr. Kritz, der mir in liebenswürdigster Weise bei allen Versuchen assistiert hat, hierfür vielmals danken.

Wurde uns auch aus äußeren Gründen nicht mehr möglich, den in weiten Rahmen angelegten Versuchsplan ersehöpfend durchzuführen, so dürften doch, bei der Einheitlichkeit der Versuchbasis, die immerhin gewonnenen Ergebnisse nicht ohne praktisches Interesse sein. 